\title{
Inventário Florístico Florestal de Santa Catarina: espécies da Floresta Estacional Decidual
}

Floristic and Forest Inventory of Santa Catarina: species of Seasonal Deciduous Forests

\author{
André Luís de Gasper ${ }^{1,5}$, Alexandre Uhlmann ${ }^{4}$, Lucia Sevegnani ${ }^{1}$, Débora Vanessa Lingner ${ }^{2}$, \\ Morilo José Rigon-Júnior ${ }^{1}$, Marcio Verdi ${ }^{1}$, Anita Stival-Santos ${ }^{1}$, Susana Dreveck ${ }^{1}$, \\ Marcos Sobral ${ }^{1} \&$ Alexander Christian Vibrans ${ }^{3}$
}

\begin{abstract}
Resumo
O presente trabalho visou apresentar e analisar a flora da Floresta Estacional Decidual em Santa Catarina, Brasil, tendo como fonte de dados os levantamentos realizados durante o Inventário Florístico Florestal de Santa Catarina. Foram avaliadas as espécies de indivíduos lenhosos de 79 unidades amostrais de $4.000 \mathrm{~m}^{2}$. Foram feitas coletas extras de indivíduos férteis, no entorno e nas unidades amostrais, das demais formas de vida. Este esforço amostral registrou 420 espécies, abrangendo 90 famílias e 275 gêneros. Nas unidades amostrais, registrou-se 233 espécies, sendo 204 com diâmetro na altura do peito (DAP) $\geq 10 \mathrm{~cm}$ e $162 \mathrm{com}$ diâmetro na altura do peito DAP $\leq 10 \mathrm{~cm}$ e altura $\geq 1,50 \mathrm{~m}$, portanto com espécies em comuns. A coleta de material extra registrou 332 angiospermas e uma gimnosperma (Araucaria angustifolia), demonstrando a importância de coletas externas às áreas previamente delimitadas. Entre as ameaçadas de extinção foram registradas Ocotea odorifera e Araucaria angustifolia.

Palavra-chave: árvores, biodiversidade, FED, IFFSC, Floresta Atlântica, riqueza.
\end{abstract}

\begin{abstract}
This paper aims at to present and analyze the flora of Seasonal Deciduous Forests in Santa Catarina, Brazil, based on results of a Floristic and Forest Inventory of Santa Catarina. We evaluated the botanical collections made in 79 sample units of $4,000 \mathrm{~m}^{2}$, within which all individual from woody species higher than $1,5 \mathrm{~m}$ were sampled. Additionally, we collected plant species in their reproductive phase both inside and outside the sample units. This sample effort registered 420 species, 90 families, and 275 genera. Inside the sample units there were 233 species, of which 204 species had a diameter at breast high $(\mathrm{DBH}) \geq 10 \mathrm{~cm} ; 162$ species with $\mathrm{DBH} \leq 10 \mathrm{~cm}$ and higher than $1,5 \mathrm{~m}$. Fertile specimens collected inside and outside the sample units included 332 angiosperms and one gymnosperm (Araucaria angustifolia), showing the importance of additional collecting. Among the endangered species we collected Ocotea odorifera and Araucaria angustifolia.
\end{abstract}

Key words: trees, biodiversity, FED, IFFSC, Atlantic Rain Forest, richness.

\section{Introdução}

O estudo da flora das florestas estacionais tropicais secas - FETS (seasonally dry tropical forests - SDTF) tem sido objeto de análise por diversos autores (Prado \& Gibbs 1993; Pennington et al. 2000; Pennington et al. 2009, LinaresPalomino 2011). Estes consideram que as flutuações climáticas ocorridas durante o Pleistoceno sejam responsáveis pela atual distribuição disjunta de FETS no neotrópico, havendo, nas condições climáticas atuais, sua retração a partir de uma cobertura pretérita mais ampla.

Segundo Gentry (1995), florestas estacionais ocorrem em regiões com precipitação atmosférica inferior a $1.600 \mathrm{~mm}$ anuais, com forte sazonalidade,

\footnotetext{
Fundação Universidade Regional de Blumenau, Herbário Dr. Roberto Miguel Klein (FURB), Depto. Ciências Naturais, R. Antônio da Veiga 140, 89012-900 Victor Konder, Blumenau, SC, Brasil.

${ }^{2}$ Universidade Federal de São João Del-Rei, Praça Frei Orlando 170, 36307-352, São João Del-Rei, MG, Brasil.

${ }^{3}$ Fundação Universidade Regional de Blumenau, Depto. Engenharia Florestal, R. Antônio da Veiga 140, 89012-900, Victor Konder, Blumenau, SC, Brasil.

${ }^{4}$ Embrapa Florestas, Est. da Ribeira, km 111, C.P. 319, 83411-000, Colombo, PR, Brasil.

${ }^{5}$ Autor para correspondencia: algasper@gmail.com
} 
havendo períodos entre cinco e seis meses com volume mensal de chuvas inferior a $100 \mathrm{~mm}$. Prado \& Gibbs (1993) apontam a existência de um "arco pleistocênico" constituído por uma extensa área de florestas sazonais que vão desde a caatinga, no nordeste brasileiro, até o sul do Brasil, inclusive parte do Paraguai e norte da Argentina, este último grupo, constituindo o "núcleo Missiones" (Prado \& Gibbs 1993; Pennington et al. 2009). Este arco agrega as mais extensas áreas de FETS em território brasileiro (Pennington et al. 2000) e é onde se insere a área de Floresta Estacional Decidual (FED) catarinense (IBGE 1992). Com relação à origem da FED, foi considerada uma vegetação recente em Santa Catarina (Bigarella 1964), sendo seu estabelecimento posterior àquele dos campos e da Floresta Ombrófila Mista, o que parece ser sustentado por Pennington et al. (2000).

A Floresta Estacional Decidual em Santa Catarina distribui-se por $7.670 \mathrm{~km}^{2} \mathrm{em} u m$ intervalo altitudinal situado entre 150 a $800 \mathrm{~m}$ (excepcionalmente $900 \mathrm{~m}$, segundo - Klein 1978) ao longo do eixo principal da bacia do rio Uruguai, irradiando-se pelos seus afluentes, onde estabelece contato com a Floresta Ombrófila Mista, em geral, a partir dos $600 \mathrm{~m}$ de altitude (Gasper et al. 2012). Por isso, sua maior expressão se dá nas porções média e baixa dos vales formados por este rio e seus afluentes, especialmente no sudoeste do estado.

Desde a segunda metade do século XX, esta região fitoecológica recebe intensa pressão de exploração dos recursos florestais e, segundo Klein (1972, 1978), à época, já sofria com a exploração indiscriminada de madeira e com a sua substituição pela agricultura, dadas as propriedades dos solos da região, tanto que as florestas primárias eram representadas por poucos e pequenos núcleos. Esta mesma condição ainda hoje prevalece, sendo a vasta maioria dos remanescentes representada por fragmentos de pequena dimensão (Ribeiro et al. 2009; Vibrans et al. 2012a; Vibrans et al. 2013). Segundo os dados de Vibrans et al. (2012a), de sua área original total, remanescem cerca de $16,3 \%$, sendo que $52 \%$ da área atualmente coberta por FED no estado está representada por fragmentos florestais com menos que 50 ha. Este dado indica que a metade da cobertura atual da FED se encontra completamente fragmentada, sendo muito raras, segundo os mesmos autores, as áreas contínuas com mais que 200 ha. A condição atual da FED é ainda mais grave se comparados os dados totais do estado, onde a soma da cobertura dos fragmentos com tamanho igual ou menor a 50 ha representa $14 \%$ da área coberta por florestas (Vibrans et al. 2012a), explicitando que a FED representa a tipologia florestal mais gravemente afetada pela colonização recente.

Em Santa Catarina, a FED foi, de forma geral, pouco estudada em termos de sua estrutura e riqueza, com exceção dos trabalhos taxonômicos desenvolvidos para a Flora Ilustrada Catarinense, que datam dos anos entre 1957 a 1964 (Reitz 1965). Diante desta carência e da exploração que esta floresta sofreu e ainda está sendo submetida, temos como perguntas: 1) quantas e quais são as espécies arbóreo-arbustivas presentes na FED atualmente; 2) o estádio sucessional avançado apresenta significante diferença florística em relação ao médio? Há algum efeito dos estádios sucessionais sobre a riqueza de espécies? 3) quantas são as espécies não encontradas por este trabalho e que foram inventariadas em trabalhos sistemáticos anteriores a este?

\section{Material e Métodos}

Coleta de dados

A relação florística aqui apresentada resulta das coletas de campo do Inventário Florístico Florestal de Santa Catarina (IFFSC), especificamente da Floresta Estacional Decidual (Fig. 1), tendo sido analisadas as espécies das 79 unidades amostrais (UA) implementadas pelo IFFSC, entre setembro de 2008 e maio de 2009 (Vibrans et al. 2010).

As precipitações anuais variam entre 1.800 a $2.000 \mathrm{~mm}$, com pequena tendência à estacionalidade (Wrege et al. 2002). Seus índices térmicos determinam dois períodos bem distintos: um de quatro a cinco meses, centrado no verão, com médias compensadas iguais ou superiores a $20^{\circ} \mathrm{C}$ e outro de dois a três meses, centrado no inverno, com médias iguais ou inferiores a $15^{\circ} \mathrm{C}$. O clima, apesar de quente-úmido durante boa parte do ano, conserva, por apreciável período, caráter frio, capaz de imprimir restrições à proliferação e ao desenvolvimento de grande número de espécies tropicais (Leite \& Klein 1990).

Ametodologia de instalação das UA foi detalhada por Vibrans et al. (2010). Cada UA inclui uma área de $4.000 \mathrm{~m}^{2}$ para o componente arbóreo, distribuídos em quatro segmentos de $20 \mathrm{~m} \times 50 \mathrm{~m}\left(1.000 \mathrm{~m}^{2}\right)$ dispostos em cruz cujos braços estão orientados na direção dos pontos cardeais, sendo no final destes braços uma parcela de $5 \mathrm{~m} \times 5 \mathrm{~m}$, totalizando $100 \mathrm{~m}^{2}$ para o componente arbustivo/subarbóreo. 


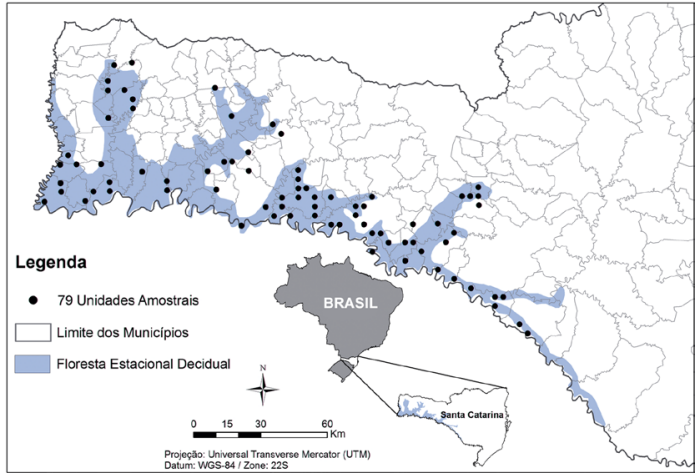

Figura 1 - Localização das 79 unidades amostrais do Inventário Florístico Florestal de Santa Catarina na região da Floresta Estacional Decidual, Santa Catarina, Brasil. Figure 1 - Localization of the 79 sample units of the Floristic and Forest Inventory of Santa Catarina state in Seasonal Deciduous Forests region, Santa Catarina, Brazil.

As coletas efetuadas durante as medições das UA neste trabalho foram divididas como (1) componente arbóreo: refere-se às espécies medidas em campo com diâmetro à altura do peito (DAP, a 1,30 m do solo) $\geq 10$ cm; (2) componente arbustivo/subarbóreo: refere-se aos indivíduos medidos em campo com DAP $\leq 10$ $\mathrm{cm}$ e altura $>1,50 \mathrm{~m}$; (3) componente florístico extra: refere-se a todas as coletas férteis feitas no interior e nos arredores das UA, mesmo que fora dos critérios de inclusão acima citados.

Apenas os indivíduos identificados em nível de espécie ou gênero foram listados. No caso da determinação ter sido possível apenas ao nível genérico, somente foram listadas aqueles representados por uma espécie, evitando assim estimativas equivocadas. A determinação do material botânico foi realizada em campo ou após coleta e herborização, mediante consulta à coleção depositada no herbário Dr. Roberto Miguel Klein, da Universidade Regional de Blumenau (FURB) e quando necessário, por meio de consulta à especialistas. Todo o material botânico coletado foi depositado no herbário FURB, e doações foram encaminhadas aos herbários BHCB, MBM e RB (siglas conforme Thiers 2012).

A circunscrição das famílias segue APG III (2009) para as angiospermas e Christenhusz et al. (2011) para as gimnospermas.

\section{Análise dos dados}

Fez-se uma comparação histórica, após sinonimização, entre a lista de espécies arbóreas apresentadas por Reitz et al. (1979) para a FED e a deste trabalho para o componente arbóreo e arbóreo/subarbóreo. As espécies amostradas pelo IFFSC, mas não listadas pelo trabalho de Reitz et al. (1979), tiveram descritas áreas de ocorrência e/ ou raridade, conforme especificado nos fascículos da Flora Ilustrada Catarinense.

As unidades amostrais foram categorizadas em estádios sucessionais (médio e avançado) conforme classificação visual de campo, análise com base na resolução CONAMA 04/94 e ajustes efetuados por Sevegnani et al. (2013). O número de espécies do componente arbóreo dos estádios (avançado e médio, valores por UA) foi comparado através do teste $T$. Antes, a homogeneidade da variância e a distribuição normal dos dados foram testadas pelos testes de Levene e KolmogorovSmirnoff, respectivamente.

Além disso, o coeficiente de correlação de Pearson foi utilizado para verificar a existência de associações entre a riqueza de espécies (somente do componente arbóreo) e variáveis relacionadas com a estrutura da floresta (dominância total - em $\mathrm{m}^{2} /$ ha e densidade total - em N ind./ha, ambos os valores calculados para toda a UA), e variáveis ambientais (altitude, latitude e longitude). Os coeficientes de correlação obtidos foram utilizados para eliminar as variáveis colineares e ajustar, através de regressão linear simples e múltipla, modelos considerando a riqueza de espécies como variável dependente. Os resíduos dos modelos foram submetidos à análise a fim de verificar a normalidade do erro.

\section{Resultados}

Considerando somente os componentes avaliados nas unidades amostrais (arbóreo e arbustivo/subarbóreo), foram registradas 233 espécies (uma gimnosperma - Araucaria angustifolia - e 232 angiospermas), das quais 133 espécies (55\%) são comuns a ambos os componentes, enquanto 71 espécies $(30 \%)$ são exclusivas do componente arbóreo e $29(12 \%)$ do componente arbustivo/subarbóreo (Tabela 2).

Considerando tanto a amostragem sistemática, quanto aquela feita nos arredores das parcelas, foram amostradas 419 espécies de angiospermas e uma gimnosperma. Esse conjunto inclui 90 famílias e 275 gêneros (Tab. 1). A família com maior riqueza específica foi Fabaceae (42 spp.), seguida de Myrtaceae (36) e Asteraceae (33). Os gêneros com maior riqueza foram Solanum L. (17 spp.), Eugenia L. (12), Peperomia Ruiz \& Pav. (8) e Myrcia DC. (7). 
Tabela 1 - Lista das espécies de angiospermas e gimnospermas coletadas durante a fase III do Inventário Florístico Florestal de Santa Catarina, na Floresta Estacional Decidual. A: coletada no componente arbóreo; R: coletada no componente arbustivo/subarbóreo; H: coletada no componente florístico. Os coletores são M. Verdi, S. Dreveck, A. Stival-Santos, A. Korte e A.L. de Gasper

Table 1 - List of angiosperms and gymnosperms collected during the phase III of the Floristic and Forest Inventory of Santa Catarina state in Seasonal Dry Forest. A: collected in tree component; R: collected in treelets and shrubs component; H: collected in floristic component. Collectors: M. Verdi, S. Dreveck, A. Stival-Santos, A. Korte and A.L. de Gasper.

\begin{tabular}{|c|c|c|c|c|c|}
\hline Família & Nome científico & $\mathbf{A}$ & $\mathbf{R}$ & $\mathbf{H}$ & Voucher \\
\hline \multirow[t]{3}{*}{ Acanthaceae } & Justicia brasiliana Roth & & $\mathrm{x}$ & $\mathrm{x}$ & Verdi 1608 \\
\hline & Justicia carnea Lindl. & & & $\mathrm{x}$ & Dreveck 796 \\
\hline & Ruellia angustiflora (Nees) Lindau ex Rambo & & $\mathrm{x}$ & & Verdi 1267 \\
\hline Achatocarpaceae & Achatocarpus praecox Griseb. & $\mathrm{x}$ & $\mathrm{x}$ & $\mathrm{x}$ & Verdi 1767 \\
\hline Adoxaceae & Sambucus australis Cham. \& Schltdl. & $\mathrm{x}$ & & $\mathrm{x}$ & Verdi 3885 \\
\hline Alstroemeriaceae & Bomarea edulis (Tussac.) Herb. & & & $\mathrm{x}$ & Gasper 1967 \\
\hline \multirow[t]{4}{*}{ Amaranthaceae } & Alternanthera micrantha R.E.Fr. & & & $\mathrm{x}$ & Verdi 2643 \\
\hline & Chamissoa acuminata Mart. & & & $\mathrm{x}$ & Stival-Santos 309 \\
\hline & Chamissoa altissima (Jacq.) Kunth & & & $\mathrm{x}$ & Stival-Santos 502 \\
\hline & Iresine diffusa Humb. \& Bonpl. ex Willd. & & & $\mathrm{x}$ & Dreveck 505 \\
\hline Amaryllidaceae & Hippeastrum reticulatum Herb. & & & $\mathrm{x}$ & Stival-Santos 547 \\
\hline \multirow[t]{3}{*}{ Anacardiaceae } & Lithrea brasiliensis Marchand & $\mathrm{x}$ & $\mathrm{x}$ & & Verdi 1167 \\
\hline & Schinus lentiscifolius Marchand & & & $\mathrm{x}$ & Verdi 993 \\
\hline & Schinus terebinthifolius Raddi & $\mathrm{x}$ & $\mathrm{x}$ & $\mathrm{x}$ & Verdi 966 \\
\hline \multirow[t]{2}{*}{ Annonaceae } & Annona emarginata (Schltdl.) H.Rainer & $\mathrm{x}$ & $\mathrm{x}$ & $\mathrm{x}$ & Gasper 1893 \\
\hline & Annona sylvatica A.St.-Hil. & $\mathrm{x}$ & $\mathrm{x}$ & $\mathrm{x}$ & Verdi 955 \\
\hline Apiaceae & Daucus pusillus Michx. & & & $\mathrm{x}$ & Verdi 972 \\
\hline \multirow[t]{7}{*}{ Apocynaceae } & Asclepias curassavica L. & & & $\mathrm{x}$ & Dreveck 601 \\
\hline & Aspidosperma australe Müll.Arg. & $\mathrm{x}$ & $\mathrm{x}$ & $\mathrm{x}$ & Verdi 1644 \\
\hline & Fischeria stellata (Vell.) E.Fourn. & & & $\mathrm{x}$ & Stival-Santos 488 \\
\hline & Oxypetalum pannosum Decne. & & & $\mathrm{x}$ & Verdi 1082 \\
\hline & Rauvolfia sellowii Müll.Arg. & $\mathrm{x}$ & $\mathrm{x}$ & $\mathrm{x}$ & Verdi 1079 \\
\hline & Schubertia sp. & & & $\mathrm{x}$ & Verdi 1665 \\
\hline & Tabernaemontana catharinensis A. DC. & $\mathrm{x}$ & & $\mathrm{x}$ & Dreveck 574 \\
\hline \multirow[t]{4}{*}{ Aquifoliaceae } & Ilex brevicuspis Reissek & $\mathrm{x}$ & & $\mathrm{x}$ & Verdi 1057 \\
\hline & Ilex microdonta Reissek & $\mathrm{x}$ & $\mathrm{x}$ & & Gasper 1915 \\
\hline & Ilex paraguariensis A. St.-Hil. & $\mathrm{x}$ & $\mathrm{x}$ & $\mathrm{x}$ & Dreveck 407 \\
\hline & Ilex theezans Mart. ex Reissek & $\mathrm{x}$ & & $\mathrm{x}$ & Verdi 1819 \\
\hline \multirow[t]{2}{*}{ Araceae } & Philodendron bipinnatifidum Schott & & & $\mathrm{x}$ & Verdi 1771 \\
\hline & Spathicarpa hastifolia Hook. & & & $\mathrm{x}$ & Verdi 1076 \\
\hline \multirow[t]{3}{*}{ Araliaceae } & Aralia warmingiana (Marchal) J.Wen & $\mathrm{x}$ & & $\mathrm{x}$ & Stival-Santos 1524 \\
\hline & Hydrocotyle quinqueloba Ruiz \& Pav. & & & $\mathrm{x}$ & Verdi 1693 \\
\hline & Schefflera morototoni (Aubl.) Maguire et al. & $\mathrm{x}$ & $\mathrm{x}$ & $\mathrm{x}$ & Dreveck 640 \\
\hline Araucariaceae & Araucaria angustifolia (Bertol.) Kuntze & $\mathrm{x}$ & $\mathrm{x}$ & $\mathrm{x}$ & Verdi 1014 \\
\hline \multirow[t]{2}{*}{ Arecaceae } & Syagrus romanzoffiana (Cham.) Glassman & $\mathrm{x}$ & $\mathrm{x}$ & & Observada em campo \\
\hline & Trithrinax brasiliensis Mart. & & $\mathrm{x}$ & & Observada em campo \\
\hline Aristolochiaceae & Aristolochia triangularis Cham. \& Schltdl. & & & $\mathrm{x}$ & Verdi 1584 \\
\hline Asparagaceae & Cordyline spectabilis Kunth \& Bouché & $\mathrm{x}$ & $\mathrm{x}$ & $\mathrm{x}$ & Dreveck 630 \\
\hline \multirow[t]{3}{*}{ Asteraceae } & Achyrocline satureioides (Lam.) DC. & & & $\mathrm{x}$ & Dreveck 653 \\
\hline & Austroeupatorium inulifolium (Kunth) R.M.King \& H.Rob. & & $\mathrm{x}$ & $\mathrm{x}$ & Verdi 1823 \\
\hline & Baccharis anomala DC. & & & $\mathrm{x}$ & Verdi 1797 \\
\hline
\end{tabular}




\begin{tabular}{|c|c|c|c|c|c|}
\hline Família & Nome científico & A & $\mathbf{R}$ & $\mathbf{H}$ & Voucher \\
\hline & Baccharis dracunculifolia $\mathrm{DC}$. & $\mathrm{x}$ & $\mathrm{x}$ & $\mathrm{x}$ & Verdi 1836 \\
\hline & Baccharis punctulata DC. & & $\mathrm{x}$ & $\mathrm{x}$ & Dreveck 576 \\
\hline & Baccharis semiserrata DC. & $\mathrm{x}$ & & & Dreveck 978 \\
\hline & Campuloclinium macrocephalum (Less.) DC. & & & $\mathrm{x}$ & Verdi 1794 \\
\hline & Chromolaena laevigata (Lam.) R.M. King \& H. Rob. & & & $\mathrm{x}$ & Verdi 1791 \\
\hline & Chromolaena odorata (L.) R.M. King \& H. Rob. & & & $\mathrm{x}$ & Verdi 1868 \\
\hline & Chromolaena pedunculosa (Hook. \& Arn.) R.M. King \& H. Rob. & & & $\mathrm{x}$ & Dreveck 678 \\
\hline & Chrysolaena platensis (Spreng.) H. Rob. & & & $\mathrm{x}$ & Stival-Santos 560 \\
\hline & Dasyphyllum spinescens (Less.) Cabrera & $\mathrm{x}$ & $\mathrm{x}$ & $\mathrm{x}$ & Verdi 1838 \\
\hline & Dasyphyllum tomentosum (Spreng.) Cabrera & $\mathrm{x}$ & & & Verdi 5382 \\
\hline & Erechtites hieraciifolia (L.) Raf. ex DC. & & & $\mathrm{x}$ & Verdi 1013 \\
\hline & Gochnatia polymorpha (Less.) Cabrera & & & $\mathrm{x}$ & Verdi 2651 \\
\hline & Jungia floribunda Less. & & & $\mathrm{x}$ & Gasper 2060 \\
\hline & Kaunia rufescens (P.W. Lund ex DC.) R.M. King \& H. Rob. & & & $\mathrm{x}$ & Dreveck 623 \\
\hline & Mikania micrantha Kunth & & & $\mathrm{x}$ & Dreveck 800 \\
\hline & Neocabreria malachophylla (Klatt) R.M. King \& H. Rob. & & $\mathrm{x}$ & $\mathrm{x}$ & Verdi 1113 \\
\hline & Piptocarpha angustifolia Dusén & $\mathrm{x}$ & $\mathrm{x}$ & & Korte 1212 \\
\hline & Pterocaulon alopecuroides (Lam.) DC. & & & $\mathrm{x}$ & Dreveck 654 \\
\hline & Pterocaulon polystachyum DC. & & & $\mathrm{x}$ & Dreveck 651 \\
\hline & Raulinoreitzia crenulata (Spreng.) R.M. King \& H. Rob. & & & $\mathrm{x}$ & Verdi 1796 \\
\hline & Senecio brasiliensis (Spreng.) Less. & & & $\mathrm{x}$ & Gasper 1901 \\
\hline & Smallanthus connatus (Spreng.) H. Rob. & & & $\mathrm{x}$ & Verdi 1839 \\
\hline & Solidago chilensis Meyen & & & $\mathrm{x}$ & Verdi 1833 \\
\hline & Trixis praestans (Vell.) Cabrera & & $\mathrm{x}$ & & Verdi 2285 \\
\hline & Urolepis hecatantha (DC.) R.M. King \& H. Rob. & & & $\mathrm{x}$ & Dreveck 605 \\
\hline & Verbesina sordescens DC. & & & $\mathrm{x}$ & Verdi 1795 \\
\hline & Vernonanthura chamaedrys (Less.) H. Rob. & & & $\mathrm{x}$ & Verdi 1798 \\
\hline & Vernonanthura discolor (Spreng.) H.Rob. & $\mathrm{x}$ & & & Gasper 919 \\
\hline & Vernonanthura montevidensis (Spreng.) H. Rob. & & & $\mathrm{x}$ & Verdi 1820 \\
\hline & Vernonanthura tweedieana (Baker) H. Rob. & & & $\mathrm{x}$ & Stival-Santos 487 \\
\hline Basellaceae & Anredera cordifolia (Ten.) Steenis & & & $\mathrm{x}$ & Stival-Santos 776 \\
\hline Begoniaceae & Begonia descoleana L.B.Sm. \& B.G. Schub. & & & $\mathrm{x}$ & Dreveck 380 \\
\hline \multirow[t]{7}{*}{ Bignoniaceae } & Adenocalymma marginatum (Cham.) DC. & & & $\mathrm{x}$ & Verdi 1670 \\
\hline & Amphilophium crucigerum (L.) L.G.Lohmann & & & $\mathrm{x}$ & Stival-Santos 708 \\
\hline & Fridericia $\mathrm{sp}$. & & & $\mathrm{x}$ & Verdi 1628 \\
\hline & Handroanthus heptaphyllus (Vell.) Mattos & $\mathrm{x}$ & & $\mathrm{x}$ & Verdi 3880 \\
\hline & Jacaranda micrantha Cham. & $\mathrm{x}$ & & $\mathrm{x}$ & Stival-Santos 508 \\
\hline & Jacaranda puberula Cham. & $\mathrm{x}$ & & $\mathrm{x}$ & Stival-Santos 302 \\
\hline & Pyrostegia venusta (Ker Gawl.) Miers & & & $\mathrm{x}$ & Dreveck 389 \\
\hline \multirow[t]{6}{*}{ Boraginaceae } & Cordia americana (L.) Gottschling \& J.S. Mill. & $\mathrm{x}$ & $\mathrm{x}$ & $\mathrm{x}$ & Stival-Santos 198 \\
\hline & Cordia ecalyculata Vell. & $\mathrm{x}$ & $\mathrm{x}$ & $\mathrm{x}$ & Verdi 1629 \\
\hline & Cordia trichotoma (Vell.) Arráb. ex Steud. & $\mathrm{x}$ & $\mathrm{x}$ & $\mathrm{x}$ & Verdi 1606 \\
\hline & Heliotropium transalpinum Vell. & & & $\mathrm{x}$ & Verdi 1616 \\
\hline & Tournefortia paniculata Cham. & & & $\mathrm{x}$ & Stival-Santos 403 \\
\hline & Varronia polycephala Lam. & $\mathrm{x}$ & & $\mathrm{x}$ & Dreveck 1358 \\
\hline \multirow[t]{3}{*}{ Bromeliaceae } & Aechmea calyculata (E. Morren) Baker & & & $\mathrm{x}$ & Stival-antos 714 \\
\hline & Aechmea recurvata (Klotzsch) L.B.Sm. & & & $\mathrm{x}$ & Stival-Santos 713 \\
\hline & Tillandsia tenuifolia $\mathrm{L}$. & & & $\mathrm{x}$ & Stival-Santos 205 \\
\hline
\end{tabular}




\begin{tabular}{|c|c|c|c|c|c|}
\hline Família & Nome científico & $\mathbf{A}$ & $\mathbf{R}$ & $\mathbf{H}$ & Voucher \\
\hline \multirow[t]{4}{*}{ Cactaceae } & Cereus sp. & $\mathrm{x}$ & & & Observada em campo \\
\hline & Lepismium warmingianum (K. Schum.) Barthlott & & & $\mathrm{x}$ & Stival-Santos 152 \\
\hline & Pereskia aculeata Mill. & & & $\mathrm{x}$ & Verdi 1661 \\
\hline & Rhipsalis baccifera (J.S. Muell.) Stearn & & & $\mathrm{x}$ & Dreveck 386 \\
\hline \multirow[t]{3}{*}{ Campanulaceae } & Lobelia exaltata Pohl & & & $\mathrm{x}$ & Dreveck 467 \\
\hline & Lobelia hassleri Zahlbr. & & & $\mathrm{x}$ & Dreveck 423 \\
\hline & Siphocampylus verticillatus G. Don & & & $\mathrm{x}$ & Stival-Santos 390 \\
\hline \multirow[t]{2}{*}{ Cannabaceae } & Celtis iguanaea (Jacq.) Sarg. & $\mathrm{x}$ & $\mathrm{x}$ & $\mathrm{x}$ & Verdi 1536 \\
\hline & Trema micrantha (L.) Blume & $\mathrm{x}$ & $\mathrm{x}$ & $\mathrm{x}$ & Verdi 1080 \\
\hline Cannaceae & Canna indica $\mathrm{L}$. & & & $\mathrm{x}$ & Verdi 1747 \\
\hline Cannelaceae & Cinnamodendron dinisii Schwacke & $\mathrm{x}$ & $\mathrm{x}$ & & Verdi 545 \\
\hline Cardiopteridaceae & Citronella paniculata (Mart.) R.A. Howard & $\mathrm{x}$ & $\mathrm{x}$ & $\mathrm{x}$ & Stival-Santos 287 \\
\hline \multirow[t]{2}{*}{ Caricaceae } & Jacaratia spinosa (Aubl.) A. DC. & $\mathrm{x}$ & & $\mathrm{x}$ & Verdi 1642 \\
\hline & Vasconcellea quercifolia A. St.-Hil. & $\mathrm{x}$ & & $\mathrm{x}$ & Verdi 1588 \\
\hline \multirow[t]{3}{*}{ Celastraceae } & Maytenus aquifolia Mart. & $\mathrm{x}$ & $\mathrm{x}$ & & Verdi 3630 \\
\hline & Pristimera celastroides (Kunth) A.C.Sm. & & & $\mathrm{x}$ & Verdi 611 \\
\hline & Schaefferia argentinensis Speg. & $\mathrm{x}$ & & $\mathrm{x}$ & Dreveck 688 \\
\hline Clethraceae & Clethra scabra Pers. & $\mathrm{x}$ & $\mathrm{x}$ & $\mathrm{x}$ & Verdi 1809 \\
\hline Combretaceae & Combretum fruticosum (Loefl.) Stuntz & & & $\mathrm{x}$ & Verdi 1812 \\
\hline \multirow[t]{4}{*}{ Commelinaceae } & Commelina villosa C.B.Clarke ex Chodat \& Hassl. & & & $\mathrm{x}$ & Gasper 1193 \\
\hline & Dichorisandra hexandra (Aubl.) Kuntze ex Hand.-Mazz & & & $\mathrm{x}$ & Gasper 1414 \\
\hline & Tradescantia fluminensis Vell. & & & $\mathrm{x}$ & Verdi 77 \\
\hline & Tripogandra diuretica (Mart.) Handlos & & & $\mathrm{x}$ & Dreveck 718 \\
\hline \multirow[t]{2}{*}{ Convolvulaceae } & Ipomoea indivisa (Vell.) Hallier f. & & & $\mathrm{x}$ & Stival-Santos 557 \\
\hline & Ipomoea quamoclit $\mathrm{L}$. & & & $\mathrm{x}$ & Verdi 1683 \\
\hline Cucurbitaceae & Melothria pendula $\mathrm{L}$. & & & $\mathrm{x}$ & Stival-Santos 649 \\
\hline Cunoniaceae & Lamanonia ternata Vell. & $\mathrm{x}$ & & & Verdi 1202 \\
\hline \multirow[t]{5}{*}{ Cyperaceae } & Carex sellowiana Schltdl. & & & $\mathrm{x}$ & Dreveck 379 \\
\hline & Cyperus andreanus Maury & & & $\mathrm{x}$ & Verdi 996 \\
\hline & Cyperus burkartii Guagl. & & & $\mathrm{x}$ & Gasper 1970 \\
\hline & Cyperus hermaphroditus (Jacq.) Standl. & & & $\mathrm{x}$ & Gasper 1977 \\
\hline & Cyperus incomtus Kunth & & & $\mathrm{x}$ & Verdi 1595 \\
\hline \multirow[t]{2}{*}{ Dioscoreaceae } & Dioscorea multiflora Mart. ex Griseb. & & & & Stival-Santos 562 \\
\hline & Dioscorea piperifolia Humb. \& Bonpl. ex Willd. & & & $\mathrm{x}$ & Verdi 977 \\
\hline Ebenaceae & Diospyros inconstans Jacq. & $\mathrm{x}$ & & & Verdi 3641 \\
\hline Elaeocarpaceae & Sloanea hirsuta (Schott) Planch. ex Benth. & $\mathrm{x}$ & & & Gasper 1432 \\
\hline \multirow[t]{2}{*}{ Erythroxylaceae } & Erythroxylum deciduum A. St.-Hil. & $\mathrm{x}$ & $\mathrm{x}$ & $\mathrm{x}$ & Dreveck 431 \\
\hline & Erythroxylum myrsinites Mart. & & & $\mathrm{x}$ & Verdi 962 \\
\hline \multirow[t]{10}{*}{ Euphorbiaceae } & Acalypha gracilis Spreng. & & $\mathrm{x}$ & $\mathrm{x}$ & Gasper 1055 \\
\hline & Actinostemon concolor (Spreng.) Müll.Arg. & $\mathrm{x}$ & $\mathrm{x}$ & $\mathrm{x}$ & Verdi 1633 \\
\hline & Alchornea sidifolia Müll.Arg. & $\mathrm{x}$ & & & Verdi 3161 \\
\hline & Alchornea triplinervia (Spreng.) Müll.Arg. & $\mathrm{x}$ & & & Verdi 2880 \\
\hline & Bernardia pulchella (Baill.) Müll. Arg. & & $\mathrm{x}$ & $\mathrm{x}$ & Verdi 1788 \\
\hline & Croton sp. & & & $\mathrm{x}$ & Stival-Santos 382 \\
\hline & Euphorbia heterophylla $\mathrm{L}$. & & & $\mathrm{x}$ & Gasper 1978 \\
\hline & Manihot grahamii Hook. & $\mathrm{x}$ & $\mathrm{x}$ & $\mathrm{x}$ & Verdi 1540 \\
\hline & Sapium glandulosum (L.) Morong & $\mathrm{x}$ & $\mathrm{x}$ & $\mathrm{x}$ & Verdi 1070 \\
\hline & Sebastiania brasiliensis Spreng. & $\mathrm{x}$ & $\mathrm{x}$ & $\mathrm{x}$ & Dreveck 641 \\
\hline
\end{tabular}




\begin{tabular}{|c|c|c|c|c|c|}
\hline Família & Nome científico & A & $\mathbf{R}$ & $\mathbf{H}$ & Voucher \\
\hline & Sebastiania commersoniana (Baill.) L.B. Sm. \& Downs & $\mathrm{x}$ & $\mathrm{X}$ & $\mathrm{x}$ & Stival-Santos 459 \\
\hline & Tetrorchidium rubrivenium Poepp. & $\mathrm{x}$ & & & Verdi 2685 \\
\hline & Tragia volubilis $\mathrm{L}$. & & & $\mathrm{x}$ & Verdi 1075 \\
\hline \multirow[t]{41}{*}{ Fabaceae } & Albizia edwallii (Hoehne) Barneby \& J.W. Grimes & $\mathrm{x}$ & $\mathrm{x}$ & $\mathrm{x}$ & Verdi 1485 \\
\hline & Albizia niopoides (Spruce ex Benth.) Burkart & $\mathrm{x}$ & $\mathrm{x}$ & & Stival-Santos 678 \\
\hline & Apuleia leiocarpa (Vogel) J.F.Macbr. & $\mathrm{x}$ & $\mathrm{x}$ & & Observada em campo \\
\hline & Ateleia glazioveana Baill. & $\mathrm{x}$ & & $\mathrm{x}$ & Dreveck 754 \\
\hline & Bauhinia forficata Link & $\mathrm{x}$ & $\mathrm{x}$ & $\mathrm{x}$ & Verdi 1604 \\
\hline & Calliandra brevipes Benth. & & & $\mathrm{x}$ & Dreveck 502 \\
\hline & Calliandra foliolosa Benth. & $\mathrm{x}$ & $\mathrm{x}$ & & Observda em campo \\
\hline & Dahlstedtia pinnata (Benth.) Malme & & $\mathrm{x}$ & $\mathrm{x}$ & Verdi 1534 \\
\hline & Dalbergia frutescens (Vell.) Britton & $\mathrm{x}$ & $\mathrm{x}$ & $\mathrm{x}$ & Gasper 1934 \\
\hline & Enterolobium contortisiliquum (Vell.) Morong & $\mathrm{x}$ & & $\mathrm{x}$ & Verdi 1664 \\
\hline & Erythrina crista-galli $\mathrm{L}$. & $\mathrm{x}$ & & & Observda em campo \\
\hline & Erythrina falcata Benth. & $\mathrm{x}$ & $\mathrm{x}$ & $\mathrm{x}$ & Verdi 1086 \\
\hline & Gleditsia amorphoides (Griseb.) Taub. & $\mathrm{x}$ & & & Observda em campo \\
\hline & Holocalyx balansae Micheli & $\mathrm{x}$ & $\mathrm{X}$ & & Korte 5564 \\
\hline & Inga edulis Mart. & $\mathrm{x}$ & & & Stival-Santos 3338 \\
\hline & Inga marginata Willd. & $\mathrm{x}$ & $\mathrm{x}$ & $\mathrm{x}$ & Stival-Santos 396 \\
\hline & Inga subnuda Salzm. ex Benth. & $\mathrm{x}$ & $\mathrm{x}$ & & Gasper 2531 \\
\hline & Inga vera Wild. & $\mathrm{x}$ & $\mathrm{x}$ & $\mathrm{x}$ & Dreveck 374 \\
\hline & Inga virescens Benth. & $\mathrm{x}$ & & & Observada em campo \\
\hline & Leptolobium elegans Vogel & $\mathrm{x}$ & & & Observada em campo \\
\hline & Lonchocarpus campestris Mart. ex Benth. & $\mathrm{x}$ & $\mathrm{x}$ & $\mathrm{x}$ & Verdi 1483 \\
\hline & Lonchocarpus cultratus (Vell.) A.M.G. Azevedo \& H.C. Lima & $\mathrm{x}$ & $\mathrm{x}$ & $\mathrm{X}$ & Verdi 1092 \\
\hline & Lonchocarpus muehlbergianus Hassl. & $\mathrm{x}$ & $\mathrm{x}$ & $\mathrm{x}$ & Stival-Santos 417 \\
\hline & Lonchocarpus nitidus (Vogel) Benth. & $\mathrm{x}$ & $\mathrm{x}$ & & Observada em campo \\
\hline & Machaerium hirtum (Vell.) Stellfeld & $\mathrm{x}$ & & & Verdi 3593 \\
\hline & Machaerium nyctitans (Vell.) Benth. & $\mathrm{x}$ & & & Korte 6991 \\
\hline & Machaerium paraguariense Hassl. & $\mathrm{x}$ & $\mathrm{x}$ & & Verdi 1337 \\
\hline & Machaerium stipitatum (DC.) Vogel & $\mathrm{x}$ & $\mathrm{x}$ & $\mathrm{x}$ & Verdi 1713 \\
\hline & Mimosa scabrella Benth. & $\mathrm{x}$ & $\mathrm{x}$ & $\mathrm{x}$ & Stival-Santos 307 \\
\hline & Myrocarpus frondosus Allemão & $\mathrm{x}$ & $\mathrm{x}$ & $\mathrm{x}$ & Verdi 3771 \\
\hline & Parapiptadenia rigida (Benth.) Brenan & $\mathrm{x}$ & $\mathrm{x}$ & $\mathrm{x}$ & Verdi 1677 \\
\hline & Peltophorum dubium (Spreng.) Taub. & $\mathrm{x}$ & & $\mathrm{x}$ & Verdi 1658 \\
\hline & Piptadenia gonoacantha (Mart.) J.F.Macbr. & $\mathrm{x}$ & & & Stival-Santos 1491 \\
\hline & Senegalia nitidifolia (Speg.) Seigler \& Ebinger & & & $\mathrm{x}$ & Dreveck 448 \\
\hline & Senegalia recurva (Benth.) Seigler \& Ebinger & $\mathrm{x}$ & $\mathrm{x}$ & $\mathrm{x}$ & Verdi 1699 \\
\hline & Senegalia tenuifolia (L.) Britton \& Rose & & & $\mathrm{x}$ & Dreveck 468 \\
\hline & Senegalia tucumanensis (Griseb.) Seigler \& Ebinger & & $\mathrm{x}$ & $\mathrm{x}$ & Stival-Santos 406 \\
\hline & Senegalia velutina (DC.) Seigler \& Ebinger & & & $\mathrm{x}$ & Stival-Santos 210 \\
\hline & Senna occidentalis (L.) Link & & & $\mathrm{x}$ & Stival-Santos 489 \\
\hline & Vicia sp. & & & $\mathrm{x}$ & Verdi 991 \\
\hline & Vigna sp. & & & $\mathrm{x}$ & Verdi 1563 \\
\hline Hypericaceae & Hypericum connatum Lam & & & $\mathrm{x}$ & Dreveck 508 \\
\hline Lamiaceae & Aegiphila brachiata Vell. & $\mathrm{x}$ & & $\mathrm{x}$ & Verdi 1026 \\
\hline & Ocimum carnosum (Spreng.) Link \& Otto ex Benth. & & & $\mathrm{x}$ & Verdi 1592 \\
\hline & Rhabdocaulon strictum (Benth.) Epling & & & $\mathrm{x}$ & Verdi 1792 \\
\hline
\end{tabular}




\begin{tabular}{|c|c|c|c|c|c|}
\hline Família & Nome científico & $\mathbf{A}$ & $\mathbf{R}$ & $\mathbf{H}$ & Voucher \\
\hline & Salvia sp. & & & $\mathrm{x}$ & Verdi 1607 \\
\hline & Vitex megapotamica (Spreng.) Moldenke & $\mathrm{x}$ & $\mathrm{x}$ & $\mathrm{x}$ & Dreveck 548 \\
\hline \multirow[t]{13}{*}{ Lauraceae } & Cinnamoтит атоепит (Nees \& Mart.) Kosterm. & $\mathrm{x}$ & $\mathrm{x}$ & & Gasper 1163 \\
\hline & Cryptocarya aschersoniana $\mathrm{Mez}$ & $\mathrm{x}$ & $\mathrm{x}$ & $\mathrm{x}$ & Dreveck 626 \\
\hline & Cryptocarya mandioccana Meisn. & $\mathrm{x}$ & & & Observada em campo \\
\hline & Endlicheria paniculata (Spreng.) J.F. Macbr. & $\mathrm{x}$ & & $\mathrm{x}$ & Stival-Santos 372 \\
\hline & Nectandra grandiflora Nees & $\mathrm{x}$ & & & Verdi 1046 \\
\hline & Nectandra lanceolata Nees & $\mathrm{x}$ & $\mathrm{x}$ & $\mathrm{x}$ & Gasper 2059 \\
\hline & Nectandra megapotamica (Spreng.) Mez & $\mathrm{x}$ & $\mathrm{x}$ & $\mathrm{x}$ & Stival-Santos 1536 \\
\hline & Nectandra membranacea (Sw.) Griseb. & $\mathrm{x}$ & $\mathrm{x}$ & & Stival-Santos 1245 \\
\hline & Ocotea diospyrifolia (Meisn.) Mez & $\mathrm{x}$ & $\mathrm{x}$ & $\mathrm{x}$ & Verdi 1643 \\
\hline & Ocotea laxa (Nees) Mez & $\mathrm{x}$ & $\mathrm{x}$ & & Stival-Santos 1521 \\
\hline & Ocotea odorifera (Vell.) Rohwer & $\mathrm{x}$ & $\mathrm{x}$ & & Verdi 3266 \\
\hline & Ocotea puberula (Rich.) Nees & $\mathrm{x}$ & $\mathrm{x}$ & $\mathrm{x}$ & Verdi 1062 \\
\hline & Ocotea pulchella (Nees \& Mart.) Mez & $\mathrm{x}$ & $\mathrm{x}$ & $\mathrm{x}$ & Verdi 544 \\
\hline Loganiaceae & Strychnos brasiliensis (Spreng.) Mart. & $\mathrm{x}$ & $\mathrm{x}$ & $\mathrm{x}$ & Gasper 1957 \\
\hline \multirow[t]{3}{*}{ Malpighiaceae } & Bunchosia maritima (Vell.) J.F. Macbr. & $\mathrm{x}$ & & $\mathrm{x}$ & Verdi 3876 \\
\hline & Dicella nucifera Chodat & & & $\mathrm{x}$ & Stival-Santos 450 \\
\hline & Heteropterys intermedia (A. Juss.) Griseb. & & & $\mathrm{x}$ & Verdi 2431 \\
\hline \multirow[t]{11}{*}{ Malvaceae } & Abutilon umbelliflorum A. St.-Hil. & & & $\mathrm{x}$ & Dreveck 381 \\
\hline & Bastardiopsis densiflora (Hook. \& Arn.) Hassl. & $\mathrm{x}$ & & $\mathrm{x}$ & Stival-Santos 705 \\
\hline & Byttneria australis A. St.-Hil. & & & $\mathrm{x}$ & Stival-Santos 208 \\
\hline & Ceiba speciosa (A. St.-Hil.) Ravenna & $\mathrm{x}$ & & $\mathrm{x}$ & Stival-Santos 451 \\
\hline & Heliocarpus popayanensis Kunth & $\mathrm{x}$ & & $\mathrm{x}$ & Verdi 2287 \\
\hline & Hibiscus sp. & & & $\mathrm{x}$ & Verdi 1755 \\
\hline & Luehea divaricata Mart. \& Zucc. & $\mathrm{x}$ & $\mathrm{x}$ & $\mathrm{x}$ & Verdi 1816 \\
\hline & Malvastrum coromandelianum Garcke & & & $\mathrm{x}$ & Dreveck 603 \\
\hline & Pavonia communis A.St.-Hil. & & & $\mathrm{x}$ & Verdi 1390 \\
\hline & Pavonia sepium A. St.-Hil. & & & $\mathrm{x}$ & Dreveck 674 \\
\hline & Sida rhombifolia $\mathrm{L}$. & & & $\mathrm{x}$ & Gasper 1936 \\
\hline Maranthaceae & Ctenanthe muelleri Petersen & & & $\mathrm{x}$ & Verdi 1101 \\
\hline \multirow[t]{5}{*}{ Melastomataceae } & Leandra australis (Cham.) Cogn. & & & $\mathrm{x}$ & Verdi 1063 \\
\hline & Leandra regnellii (Triana) Cogn. & & & $\mathrm{x}$ & Stival-Santos 151 \\
\hline & Leandra xanthocoma (Naudin) Cogn. & & & $\mathrm{x}$ & Verdi 1011 \\
\hline & Miconia cinerascens Miq. & $\mathrm{x}$ & $\mathrm{x}$ & $\mathrm{x}$ & Verdi 1053 \\
\hline & Miconia pusilliflora (DC.) Naudin & & & $\mathrm{x}$ & Verdi 1784 \\
\hline \multirow[t]{6}{*}{ Meliaceae } & Cabralea canjerana (Vell.) Mart. & $\mathrm{x}$ & $\mathrm{x}$ & $\mathrm{x}$ & Stival-Santos 429 \\
\hline & Cedrela fissilis Vell. & $\mathrm{x}$ & $\mathrm{x}$ & $\mathrm{x}$ & Verdi 1564 \\
\hline & Guarea macrophylla Vahl & $\mathrm{x}$ & $\mathrm{x}$ & $\mathrm{x}$ & Verdi 1090 \\
\hline & Trichilia catigua A. Juss. & $\mathrm{x}$ & $\mathrm{x}$ & $\mathrm{x}$ & Dreveck 716 \\
\hline & Trichilia claussenii C. DC. & $\mathrm{x}$ & $\mathrm{x}$ & $\mathrm{x}$ & Verdi 1641 \\
\hline & Trichilia elegans A. Juss. & $\mathrm{x}$ & $\mathrm{x}$ & $\mathrm{x}$ & Verdi 1587 \\
\hline Monimiaceae & Hennecartia omphalandra J.Poiss. & $\mathrm{x}$ & $\mathrm{x}$ & $\mathrm{x}$ & Verdi 968 \\
\hline \multirow[t]{5}{*}{ Moraceae } & Ficus adhatodifolia Schott & $\mathrm{x}$ & & & Stival-Santos 1059 \\
\hline & Ficus gomelleira Kunth & $\mathrm{x}$ & & & Dreveck 2543 \\
\hline & Ficus luschnathiana (Miq.) Miq. & $\mathrm{x}$ & $\mathrm{x}$ & $\mathrm{x}$ & Verdi 1782 \\
\hline & Maclura tinctoria (L.) D. Don ex Steud. & $\mathrm{x}$ & & $\mathrm{x}$ & Verdi 1639 \\
\hline & Sorocea bonplandii (Baill.) W.C.Burger et al. & $\mathrm{x}$ & $\mathrm{x}$ & & Korte 1206 \\
\hline
\end{tabular}




\begin{tabular}{|c|c|c|c|c|c|}
\hline Família & Nome científico & $\mathbf{A}$ & $\mathbf{R}$ & $\mathbf{H}$ & Voucher \\
\hline \multirow[t]{34}{*}{ Myrtaceae } & Acca sellowiana (O. Berg) Burret & & & $\mathrm{x}$ & Verdi 987 \\
\hline & Blepharocalyx salicifolius (Kunth) O.Berg & $\mathrm{x}$ & & & Verdi 1183 \\
\hline & Calyptranthes grandifolia O.Berg & $\mathrm{x}$ & $\mathrm{x}$ & & Verdi 2579 \\
\hline & Calyptranthes tricona D. Legrand & $\mathrm{x}$ & $\mathrm{x}$ & $\mathrm{x}$ & Dreveck 912 \\
\hline & Campomanesia guaviroba (DC.) Kiaersk. & $\mathrm{x}$ & & & Stival-Santos 248 \\
\hline & Campomanesia guazumifolia (Cambess.) O. Berg & $\mathrm{x}$ & $\mathrm{x}$ & $\mathrm{x}$ & Stival-Santos 351 \\
\hline & Campomanesia xanthocarpa (Mart.) O.Berg & $\mathrm{x}$ & $\mathrm{x}$ & $\mathrm{x}$ & Verdi 945 \\
\hline & Eugenia burkartiana (D. Legrand) D. Legrand & $\mathrm{x}$ & $\mathrm{x}$ & $\mathrm{x}$ & Verdi 1593 \\
\hline & Eugenia gracillima Kiaersk. & $\mathrm{x}$ & & $\mathrm{x}$ & Dreveck 1888 \\
\hline & Eugenia hiemalis Cambess. & & $\mathrm{x}$ & & Verdi 2682 \\
\hline & Eugenia involucrata DC. & $\mathrm{x}$ & $\mathrm{x}$ & & Stival-Santos 2058 \\
\hline & Eugenia pyriformis Cambess. & $\mathrm{x}$ & $\mathrm{x}$ & $\mathrm{x}$ & Stival-Santos 310 \\
\hline & Eugenia ramboi D. Legrand & $\mathrm{x}$ & $\mathrm{x}$ & $\mathrm{x}$ & Gasper 1897 \\
\hline & Eugenia rostrifolia D. Legrand & $\mathrm{x}$ & $\mathrm{x}$ & & Korte 6258 \\
\hline & Eugenia subterminalis DC. & $\mathrm{x}$ & & & Gasper 917 \\
\hline & Eugenia uniflora L. & $\mathrm{x}$ & $\mathrm{x}$ & & Dreveck 513 \\
\hline & Eugenia uruguayensis Cambess. & $\mathrm{x}$ & & & Verdi 1409 \\
\hline & Eugenia verticillata (Vell.) Angely & $\mathrm{x}$ & $\mathrm{x}$ & $\mathrm{x}$ & Dreveck 608 \\
\hline & Myrceugenia myrcioides (Cambess.) O.Berg & $\mathrm{x}$ & $\mathrm{x}$ & & Verdi 2581 \\
\hline & Myrcia guianensis (Aubl.) DC. & & & $\mathrm{x}$ & Stival-Santos 299 \\
\hline & Myrcia hebepetala DC. & $\mathrm{x}$ & & & Dreveck 899 \\
\hline & Myrcia oblongata DC. & $\mathrm{x}$ & $\mathrm{x}$ & $\mathrm{x}$ & Verdi 1793 \\
\hline & Myrcia palustris DC. & & $\mathrm{x}$ & $\mathrm{x}$ & Verdi 1817 \\
\hline & Myrcia pubipetala Miq. & $\mathrm{x}$ & $\mathrm{x}$ & & Gasper 2233 \\
\hline & Myrcia selloi (Spreng.) N.Silveira & & $\mathrm{x}$ & & Verdi 3889 \\
\hline & Myrcia splendens (Sw.) DC. & $\mathrm{x}$ & & & Gasper 1580 \\
\hline & Myrcianthes gigantea (D.Legrand) D.Legrand & $\mathrm{x}$ & & & Gasper 2506 \\
\hline & Myrcianthes pungens (O.Berg) D.Legrand & $\mathrm{x}$ & $\mathrm{x}$ & & Verdi 1182 \\
\hline & Myrciaria floribunda (H. West ex Willd.) O. Berg & $\mathrm{x}$ & $\mathrm{x}$ & $\mathrm{x}$ & Dreveck 492 \\
\hline & Myrrhinium atropurpureum Schott & & $\mathrm{x}$ & & Gasper 1447 \\
\hline & Plinia rivularis (Cambess.) Rotman & $\mathrm{x}$ & $\mathrm{x}$ & $\mathrm{x}$ & Stival-Santos 2059 \\
\hline & Plinia trunciflora (O.Berg) Kausel & $\mathrm{x}$ & $\mathrm{x}$ & & Gasper 2530 \\
\hline & Psidium australe Cambess. & & & $\mathrm{x}$ & Verdi 1811 \\
\hline & Siphoneugena reitzii D.Legrand & $\mathrm{x}$ & & & Verdi 2062 \\
\hline Nyctaginaceae & Pisonia ambigua Heimerl & $\mathrm{x}$ & & $\mathrm{x}$ & Verdi 1744 \\
\hline Oleaceae & Chionanthus trichotomus (Vell.) P.S.Green & $\mathrm{x}$ & & & Gasper 2498 \\
\hline Onagraceae & Oenothera sp. & & & $\mathrm{x}$ & Verdi 967 \\
\hline Opiliaceae & Agonandra excelsa Griseb. & $\mathrm{x}$ & & $\mathrm{x}$ & Verdi 3875 \\
\hline \multirow[t]{8}{*}{ Orchidaceae } & Alatiglossum longipes (Lindl.) Baptista & & & $\mathrm{x}$ & Dreveck 661 \\
\hline & Baptistonia riograndensis (Cogn.) Chiron \& V.P.Castro & & & $\mathrm{x}$ & Dreveck 664 \\
\hline & Corymborkis flava (Sw.) Kuntze & & & $\mathrm{x}$ & Dreveck 647 \\
\hline & Galeandra beyrichii Rchb. f. & & & $\mathrm{x}$ & Stival-Santos 282 \\
\hline & Gomesa recurva $\mathrm{R} . \mathrm{Br}$. & & & $\mathrm{x}$ & Verdi 1527 \\
\hline & Govenia urticulata (Sw.) Lindl. & & & $\mathrm{x}$ & Verdi 1886 \\
\hline & Grandiphyllum divaricatum (Lindl.) Docha Neto & & & $\mathrm{x}$ & Dreveck 418 \\
\hline & Mesadenella cuspidata (Lindl.) Garay & & & $\mathrm{x}$ & Verdi 1720 \\
\hline Oxalidaceae & Oxalis cytisoides Mart. ex Zucc. & & & $\mathrm{x}$ & Verdi 986 \\
\hline Passifloraceae & Passiflora amethystina J.C. Mikan & & & $\mathrm{x}$ & Verdi 1832 \\
\hline
\end{tabular}




\begin{tabular}{|c|c|c|c|c|c|}
\hline Família & Nome científico & $\mathbf{A}$ & $\mathbf{R}$ & $\mathbf{H}$ & Voucher \\
\hline & Passiflora caerulea $\mathrm{L}$. & & & $\mathrm{x}$ & Stival-Santos 703 \\
\hline & Passiflora capsularis L. & & & $\mathrm{x}$ & Dreveck 454 \\
\hline \multirow[t]{5}{*}{ Phytolaccaceae } & Phytolacca americana $\mathrm{L}$. & & & $\mathrm{x}$ & Dreveck 611 \\
\hline & Phytolacca dioica $\mathrm{L}$. & $\mathrm{x}$ & & $\mathrm{x}$ & Dreveck 402 \\
\hline & Seguieria aculeata Jacq. & $\mathrm{x}$ & $\mathrm{x}$ & $\mathrm{x}$ & Verdi 1749 \\
\hline & Seguieria americana $\mathrm{L}$. & & & $\mathrm{x}$ & Dreveck 624 \\
\hline & Seguieria langsdorffii Moq. & $\mathrm{x}$ & & & Gasper 2500 \\
\hline Picramniaceae & Picramnia parvifolia Engl. & $\mathrm{x}$ & & $\mathrm{x}$ & Verdi 1068 \\
\hline \multirow[t]{15}{*}{ Piperaceae } & Peperomia blanda (Jacq.) Kunth & & & $\mathrm{x}$ & Verdi 1662 \\
\hline & Peperomia corcovadensis Gardner. & & & $\mathrm{x}$ & Gasper 1904 \\
\hline & Peperomia delicatula Henschen & & & $\mathrm{x}$ & Gasper 2065 \\
\hline & Peperomia pereskiifolia (Jacq.) Kunth & & & $\mathrm{x}$ & Stival-Santos 296 \\
\hline & Peperomia tetraphylla (G. Forst.) Hook. \& Arn. & & & $\mathrm{x}$ & Stival-Santos 648 \\
\hline & Peperomia trineura Miq. & & & $\mathrm{x}$ & Dreveck 639 \\
\hline & Peperomia trineuroides Dahlst. & & & $\mathrm{x}$ & Verdi 1087 \\
\hline & Peperomia urocarpa Fisch. \& C.A. Mey. & & & $\mathrm{x}$ & Verdi 1486 \\
\hline & Piper aduncum L. & & $\mathrm{x}$ & $\mathrm{x}$ & Dreveck 444 \\
\hline & Piper amalago L. & & $\mathrm{x}$ & $\mathrm{x}$ & Verdi 1750 \\
\hline & Piper caldense C.DC. & & $\mathrm{x}$ & & Dreveck 1650 \\
\hline & Piper gaudichaudianum Kunth & & & $\mathrm{x}$ & Verdi 1638 \\
\hline & Piper hispidum $\mathrm{Sw}$. & & $\mathrm{x}$ & & Verdi 1505 \\
\hline & Piper macedoi Yunck. & & & $\mathrm{x}$ & Dreveck 408 \\
\hline & Piper mikanianum (Kunth) Steud. & & & $\mathrm{x}$ & Verdi 946 \\
\hline \multirow[t]{2}{*}{ Plantaginaceae } & Plantago sp. & & & $\mathrm{x}$ & Gasper 1980 \\
\hline & Stemodia verticillata (Mill.) Hassl. & & & $\mathrm{x}$ & Verdi 1557 \\
\hline \multirow[t]{13}{*}{ Poaceae } & Andropogon bicornis L. & & & $\mathrm{x}$ & Dreveck 659 \\
\hline & Eustachys distichophylla (Lag.) Nees & & & $\mathrm{x}$ & Verdi 1567 \\
\hline & Lasiacis divaricata (L.) Hitch. & & & $\mathrm{x}$ & Verdi 1735 \\
\hline & Melica macra Ness & & & $\mathrm{x}$ & Verdi 983 \\
\hline & Merostachys skvortzovii Send. & & & $\mathrm{x}$ & Verdi 969 \\
\hline & Olyra humilis Nees & & & $\mathrm{x}$ & Verdi 1035 \\
\hline & Oplismenus hirtellus (L.) P. Beauv. & & & $\mathrm{x}$ & Stival-Santos 482 \\
\hline & Pharus latifolius L. & & & $\mathrm{x}$ & Verdi 1730 \\
\hline & Pseudechinolaena polystachya (Kunth) Stapf & & & $\mathrm{x}$ & Gasper 1981 \\
\hline & Schizachyrium microstachyum (Desv. ex Ham.) Roseng. & & & $\mathrm{x}$ & Verdi 1871 \\
\hline & Setaria parviflora (Poir.) Kerguélen & & & $\mathrm{x}$ & Verdi 1003 \\
\hline & Setaria vulpiseta (Lam.) Roem. \& Schult. & & & $\mathrm{x}$ & Dreveck 625 \\
\hline & Sporobolus pseudairoides Parodi & & & $\mathrm{x}$ & Gasper 1971 \\
\hline \multirow[t]{3}{*}{ Polygonaceae } & Coccoloba argentinensis Speg. & $\mathrm{x}$ & & $\mathrm{x}$ & Stival-Santos 2057 \\
\hline & Polygonum punctatum Elliott & & & $\mathrm{x}$ & Verdi 956 \\
\hline & Ruprechtia laxiflora Meisn. & $\mathrm{x}$ & $\mathrm{x}$ & $\mathrm{x}$ & Stival-Santos 1802 \\
\hline \multirow[t]{6}{*}{ Primulaceae } & Myrsine balansae (Mez) Arechav. & & $\mathrm{x}$ & $\mathrm{x}$ & Stival-Santos 710 \\
\hline & Myrsine coriacea (Sw.) R. Br. ex Roem. \& Schult. & $\mathrm{x}$ & $\mathrm{x}$ & $\mathrm{x}$ & Verdi 1061 \\
\hline & Myrsine guianensis (Aubl.) Kuntze & $\mathrm{x}$ & $\mathrm{x}$ & $\mathrm{x}$ & Verdi 1882 \\
\hline & Myrsine loefgrenii (Mez) Imkhan. & $\mathrm{x}$ & $\mathrm{x}$ & & Dreveck 1948 \\
\hline & Myrsine parvula $(\mathrm{Mez})$ Otegui & & $\mathrm{x}$ & & Verdi 2339 \\
\hline & Myrsine umbellata Mart. & $\mathrm{x}$ & $\mathrm{x}$ & $\mathrm{x}$ & Gasper 2054 \\
\hline Proteaceae & Roupala montana Aubl. & $\mathrm{x}$ & $\mathrm{x}$ & & Verdi 1169 \\
\hline
\end{tabular}




\begin{tabular}{|c|c|c|c|c|c|}
\hline Família & Nome científico & $\mathbf{A}$ & $\mathbf{R}$ & $\mathbf{H}$ & Voucher \\
\hline Quillajaceae & Quillaja brasiliensis (A.St.-Hil. \& Tul.) Mart. & $\mathrm{x}$ & & & Verdi 198 \\
\hline \multirow[t]{2}{*}{ Rosaceae } & Prunus myrtifolia (L.) Urb. & $\mathrm{x}$ & $\mathrm{x}$ & $\mathrm{x}$ & Dreveck 620 \\
\hline & Rubus brasiliensis Mart. & & & $\mathrm{x}$ & Verdi 1384 \\
\hline \multirow[t]{14}{*}{ Rubiaceae } & Cordiera concolor (Cham.) Kuntze & $\mathrm{x}$ & & & Gasper 1266 \\
\hline & Coussarea contracta (Walp.) Müll. Arg. & $\mathrm{x}$ & $\mathrm{x}$ & $\mathrm{x}$ & Dreveck 613 \\
\hline & Coutarea hexandra (Jacq.) K. Schum. & $\mathrm{x}$ & $\mathrm{x}$ & $\mathrm{x}$ & Verdi 1815 \\
\hline & Galium sp. & & & $\mathrm{x}$ & Verdi 1031 \\
\hline & Manettia luteo-rubra (Vell.) Benth. & & & $\mathrm{x}$ & Dreveck 801 \\
\hline & Manettia paraguariensis Chodat & & & $\mathrm{x}$ & Verdi 1696 \\
\hline & Mitracarpus sp. & & & $\mathrm{x}$ & Gasper 2151 \\
\hline & Palicourea australis C.M. Taylor & & & $\mathrm{x}$ & Dreveck 405 \\
\hline & Psychotria carthagenensis Jacq. & & $\mathrm{x}$ & $\mathrm{x}$ & Verdi 1579 \\
\hline & Psychotria leiocarpa Cham. \& Schltdl. & & $\mathrm{x}$ & $\mathrm{x}$ & Verdi 1045 \\
\hline & Psychotria myriantha Müll.Arg. & & & $\mathrm{x}$ & Stival-Santos 651 \\
\hline & Psychotria suterella Müll.Arg. & & & $\mathrm{x}$ & Dreveck 619 \\
\hline & Randia ferox (Cham. \& Schltdl.) DC. & $\mathrm{x}$ & $\mathrm{x}$ & $\mathrm{x}$ & Verdi 1528 \\
\hline & Rudgea parquioides (Cham.) Müll. Arg. & & & $\mathrm{x}$ & Stival-Santos 377 \\
\hline \multirow[t]{7}{*}{ Rutaceae } & Balfourodendron riedelianum (Engl.) Engl. & $\mathrm{x}$ & $\mathrm{x}$ & $\mathrm{x}$ & Verdi 1598 \\
\hline & Esenbeckia grandiflora Mart. & $\mathrm{x}$ & $\mathrm{x}$ & $\mathrm{x}$ & Stival-Santos 433 \\
\hline & Helietta apiculata Benth. & $\mathrm{x}$ & $\mathrm{x}$ & $\mathrm{x}$ & Verdi 1524 \\
\hline & Pilocarpus pennatifolius Lem. & $\mathrm{x}$ & $\mathrm{x}$ & $\mathrm{x}$ & Verdi 1824 \\
\hline & Zanthoxylum fagara (L.) Sarg. & $\mathrm{x}$ & $\mathrm{x}$ & $\mathrm{x}$ & Verdi 1764 \\
\hline & Zanthoxylum petiolare A. St.-Hil. \& Tul. & $\mathrm{x}$ & $\mathrm{x}$ & $\mathrm{x}$ & Verdi 1799 \\
\hline & Zanthoxylum rhoifolium Lam. & $\mathrm{x}$ & $\mathrm{x}$ & $\mathrm{x}$ & Stival-Santos 556 \\
\hline \multirow[t]{5}{*}{ Salicaceae } & Banara tomentosa Clos & $\mathrm{x}$ & $\mathrm{x}$ & $\mathrm{x}$ & Verdi 1481 \\
\hline & Casearia decandra Jacq. & $\mathrm{x}$ & $\mathrm{x}$ & $\mathrm{x}$ & Stival-Santos 286 \\
\hline & Casearia obliqua Spreng. & $\mathrm{x}$ & & & Verdi 1350 \\
\hline & Casearia sylvestris $\mathrm{Sw}$. & $\mathrm{x}$ & $\mathrm{x}$ & $\mathrm{x}$ & Gasper 1899 \\
\hline & Xylosma pseudosalzmannii Sleumer & $\mathrm{x}$ & & & Gasper 987 \\
\hline \multirow[t]{13}{*}{ Sapindaceae } & Allophylus edulis (A.St.-Hil. et al.) Hieron. ex Niederl. & $\mathrm{x}$ & $\mathrm{x}$ & $\mathrm{x}$ & Dreveck 413 \\
\hline & Allophylus guaraniticus (A.St.-Hil.) Radlk. & $\mathrm{x}$ & $\mathrm{x}$ & $\mathrm{x}$ & Verdi 1772 \\
\hline & Allophylus petiolulatus Radlk. & $\mathrm{x}$ & & & Stival-Santos 898 \\
\hline & Allophylus puberulus (Cambess.) Radlk & $\mathrm{x}$ & $\mathrm{x}$ & $\mathrm{x}$ & Verdi 982 \\
\hline & Cardiospermum halicacabum L. & & & $\mathrm{x}$ & Stival-Santos 506 \\
\hline & Cupania vernalis Cambess. & $\mathrm{x}$ & $\mathrm{x}$ & $\mathrm{x}$ & Verdi 959 \\
\hline & Diatenopteryx sorbifolia Radlk. & $\mathrm{x}$ & $\mathrm{x}$ & & Dreveck 2855 \\
\hline & Matayba elaeagnoides Radlk. & $\mathrm{x}$ & $\mathrm{x}$ & $\mathrm{x}$ & Verdi 1105 \\
\hline & Matayba intermedia Radlk. & $\mathrm{x}$ & & & Verdi 3272 \\
\hline & Paullinia meliifolia Juss. & & & $\mathrm{x}$ & Stival-Santos 495 \\
\hline & Serjania sp. & & & $\mathrm{x}$ & Verdi 1346 \\
\hline & Thinouia sp. & & & & Stival-Santos 1534 \\
\hline & Urvillea sp. & & & $\mathrm{x}$ & Gasper 1451 \\
\hline \multirow[t]{3}{*}{ Sapotaceae } & Chrysophyllum gonocarpum (Mart. \& Eichler ex Miq.) Engl. & $\mathrm{x}$ & $\mathrm{x}$ & $\mathrm{x}$ & Verdi 1085 \\
\hline & Chrysophyllum inornatum Mart. & $\mathrm{x}$ & $\mathrm{x}$ & & Gasper 1512 \\
\hline & Chrysophyllum marginatum (Hook. \& Arn.) Radlk. & $\mathrm{x}$ & $\mathrm{x}$ & $\mathrm{x}$ & Verdi 1681 \\
\hline Simaroubaceae & Picrasma crenata (Vell.) Engl. & $\mathrm{x}$ & $\mathrm{x}$ & $\mathrm{x}$ & Dreveck 451 \\
\hline \multirow[t]{2}{*}{ Solanaceae } & Aureliana sp. & & $\mathrm{x}$ & $\mathrm{x}$ & Verdi 961 \\
\hline & Capsicum flexuosum Sendtn. & & & $\mathrm{x}$ & Dreveck 403 \\
\hline
\end{tabular}




\begin{tabular}{|c|c|c|c|c|c|}
\hline Família & Nome científico & $\mathbf{A}$ & $\mathbf{R}$ & $\mathbf{H}$ & Voucher \\
\hline & Cestrum intermedium Sendtn. & $\mathrm{x}$ & $\mathrm{x}$ & & Gasper 1259 \\
\hline & Cestrum strigilatum Ruiz \& Pav. & & $\mathrm{x}$ & $\mathrm{x}$ & Verdi 1558 \\
\hline & Nicotiana alata Link \& Otto & & & $\mathrm{x}$ & Verdi 970 \\
\hline & Sessea regnellii Taub. & $\mathrm{x}$ & $\mathrm{x}$ & & Gasper 1706 \\
\hline & Solanum americanum Mill. & & & $\mathrm{x}$ & Verdi 3776 \\
\hline & Solanum bullatum Vell. & $\mathrm{x}$ & $\mathrm{x}$ & & Korte 1446 \\
\hline & Solanum compressum L.B.Sm. \& Downs & $\mathrm{x}$ & & & Gasper 1398 \\
\hline & Solanum corymbiflorum (Sendtn.) Bohs & & & $\mathrm{x}$ & Dreveck 404 \\
\hline & Solanum diploconos (Mart.) Bohs & & & $\mathrm{x}$ & Verdi 1651 \\
\hline & Solanum fusiforme L.B. Sm. \& Downs & & & $\mathrm{x}$ & Stival-Santos 384 \\
\hline & Solanum guaraniticum A. St.-Hil. & & & $\mathrm{x}$ & Verdi 1016 \\
\hline & Solanum hirtellum (Spreng.) Hassl. & & & $\mathrm{x}$ & Verdi 1627 \\
\hline & Solanum mauritianum Scop. & $\mathrm{x}$ & $\mathrm{x}$ & $\mathrm{x}$ & Stival-Santos 303 \\
\hline & Solanum pabstii L.B.Sm. \& Downs & & $\mathrm{x}$ & & Dreveck 338 \\
\hline & Solanum pseudocapsicum L. & & & $\mathrm{x}$ & Stival-Santos 156 \\
\hline & Solanum pseudoquina A.St.-Hil. & $\mathrm{x}$ & & & Stival-Santos 181 \\
\hline & Solanum sanctaecatharinae Dunal & $\mathrm{x}$ & $\mathrm{x}$ & $\mathrm{x}$ & Verdi 1635 \\
\hline & Solanum sisymbriifolium Lam. & & & $\mathrm{x}$ & Dreveck 500 \\
\hline & Solanum trachytrichium Bitter & & & $\mathrm{x}$ & Stival-Santos 202 \\
\hline & Solanum vaillantii Dunal & & & $\mathrm{x}$ & Gasper 1898 \\
\hline & Solanum variabile Mart. & & $\mathrm{x}$ & $\mathrm{x}$ & Verdi 1386 \\
\hline & Vassobia breviflora (Sendtn.) Hunz. & & & $\mathrm{x}$ & Dreveck 610 \\
\hline Styracaceae & Styrax leprosus Hook. \& Arn. & $\mathrm{x}$ & $\mathrm{x}$ & $\mathrm{x}$ & Verdi 1653 \\
\hline \multirow[t]{2}{*}{ Symplocaceae } & Symplocos tetrandra Mart. & $\mathrm{x}$ & & & Verdi 1932 \\
\hline & Symplocos uniflora (Pohl) Benth. & $\mathrm{x}$ & & & Verdi 1165 \\
\hline Talinaceae & Talinum paniculatum (Jacq.) Gaertn. & & & $\mathrm{x}$ & Gasper 2150 \\
\hline Thymelaeaceae & Daphnopsis racemosa Griseb. & & $\mathrm{x}$ & & Verdi 3772 \\
\hline \multirow[t]{4}{*}{ Urticaceae } & Boehmeria caudata $\mathrm{Sw}$ & & $\mathrm{x}$ & $\mathrm{x}$ & Verdi 2633 \\
\hline & Coussapoa microcarpa (Schott) Rizzini & $\mathrm{x}$ & & & Verdi 1376 \\
\hline & Pilea pubescens Liebm. & & & $\mathrm{x}$ & Verdi 1731 \\
\hline & Urera baccifera (L.) Gaudich. ex Wedd. & $\mathrm{x}$ & $\mathrm{x}$ & $\mathrm{x}$ & Gasper 1975 \\
\hline Valerianaceae & Valeriana scandens $\mathrm{L}$. & & & $\mathrm{x}$ & Verdi 1081 \\
\hline \multirow[t]{7}{*}{ Verbenaceae } & Aloysia virgata (Ruiz \& Pav.) Juss. & $\mathrm{x}$ & $\mathrm{x}$ & $\mathrm{x}$ & Verdi 1556 \\
\hline & Citharexylum solanaceum Cham. & & & $\mathrm{x}$ & Verdi 1383 \\
\hline & Lantana camara $\mathrm{L}$. & & $\mathrm{x}$ & $\mathrm{x}$ & Verdi 985 \\
\hline & Lippia brasiliensis (Link) T.R.S. Silva & & & $\mathrm{x}$ & Dreveck 677 \\
\hline & Lippia lippioides (Cham.) Rusby & & & $\mathrm{x}$ & Dreveck 510 \\
\hline & Verbena bonariensis $\mathrm{L}$. & & & $\mathrm{x}$ & Dreveck 438 \\
\hline & Verbena litoralis Kunth & & & $\mathrm{x}$ & Verdi 1724 \\
\hline Violaceae & Hybanthus bigibbosus (A. St.-Hil.) Hassl. & $\mathrm{x}$ & $\mathrm{x}$ & $\mathrm{x}$ & Verdi 1159 \\
\hline \multirow[t]{2}{*}{ Vitaceae } & Cissus sulcicaulis (Baker) Planch. & & & $\mathrm{x}$ & Dreveck 643 \\
\hline & Cissus verticillata (L.) Nicolson \& C. E. Jarvis & & & $\mathrm{x}$ & Dreveck 507 \\
\hline Vivianiaceae & Caesarea albiflora Cambess. & & & $\mathrm{x}$ & Verdi 1829 \\
\hline
\end{tabular}


Tabela 2 - Síntese da florística amostrada na Floresta Estacional Decidual e seus ecótonos com a Floresta Ombrófila Mista e Estepe Ombrófila, no Inventário Florístico Florestal de Santa Catarina.

Table 2 - Summary of the sampled species within the Seasonal Deciduous Forest and its ecotones with the Mixed Ombrophilous Forest and grasslands in the Floristic and Forest Inventory of Santa Catarina State.

\begin{tabular}{lc}
\hline Florística do Inventário Florestal de Santa Catarina & $\mathbf{n}^{\mathbf{0}}$ \\
\hline \hline Famílias de angiospermas & 89 \\
Famílias de angiospermas com 10 ou mais espécies & 11 \\
Gêneros de angiospermas & 275 \\
Espécies amostradas & 420 \\
Espécies de angiospermas & 419 \\
Espécies do componente arbóreo & 204 \\
Espécies do componente arbustivo/subarbóreo, mas não & 29 \\
no arbóreo & \\
Espécies do componente arbóreo, não amostradas no & 71 \\
arbustivo/subarbóreo & \\
Espécies exclusivamente levantadas na florística de & 187 \\
angiospermas & \\
Espécies na lista das ameaçadas de extinção MMA-2008 & 2 \\
Espécies arbóreas citadas por Reitz et al. (1979) não & 37 \\
\hline encontradas no IFFSC & \\
\hline
\end{tabular}

Foram registradas as seguintes espécies exóticas: Citrus reticulata Blanco, Citrus x limon (L.) Burm. f., Hovenia dulcis Thunb., Morus nigra L. e Persea americana Mill. dentro das parcelas do componente arbóreo e arbustivo/subarbóreo. Contudo, estas espécies não foram contabilizadas na contagem de espécies. Cabe destaque que Meyer et al. (2012) registrou outras 20 espécies exóticas para esta região fitoecológica.

O número médio de espécies no componente arbóreo foi de 36,45 (com mínimo de 12 e máximo de 59) e no componente arbustivo/subarbóreo 14,85 (com mínimo de quatro e máximo de 32).

A coleta de material fértil (componente florístico extra) registrou 332 angiospermas e uma gimnosperma A. angustifolia (Tab. 2). Das 333 espécies, 190 são árvores e arbustos (sendo que 115 não foram registrados dentro das parcelas), 77 ervas terrícolas, 19 espécies de epífitos e 42 lianas.

Dentre as 164 espécies arbóreas citadas por Reitz et al. (1979) para a região fitoecológica, não foram amostradas 36 espécies (Tab. 3). Foram registradas ainda as espécies Araucaria angustifolia e Ocotea odorifera como ameaçadas de extinção (MMA 2008).

Considerando somente o componente arbóreo, as zonas proximais ao contato com a Floresta Ombrófila Mista, nas regiões mais elevadas ao norte e leste, se caracterizam pela maior riqueza de espécies, conforme demonstrado pela relação significativa da regressão múltipla $\left(\mathrm{R}^{2}\right.$ múltiplo $=0,37 ; \mathrm{p}<0,01)$ entre a riqueza de espécies (variável dependente) e as variáveis longitude e altitude (variáveis independentes), o que pode ser deduzido também através da Fig. 2.

Os dados de riqueza de espécies, segundo os testes aplicados, atenderam às premissas do teste $T$ quanto à normalidade e homocedasticidade. Considerando o componente arbóreo, a análise atestou haver maior riqueza de espécies em áreas de estágio avançado (42,4 espécies/UA) em relação às áreas de estágio médio (35,9 espécies/UA; $\mathrm{p}<0,01)$. (Fig. 3a). A análise de correlação entre os dados de riqueza de espécies arbóreas e a dominância total de cada UA demonstrou haver uma potencial relação positiva e significativa $(\mathrm{R}=0,43, \mathrm{p}=4,4 \times$ $10^{-5}$, Fig. 3b).

\section{Discussão}

As coletas do IFFSC contemplaram 61,8\% das espécies relacionadas por Stehmann et al. (2009) para a FED no Domínio da Floresta Atlântica brasileira. Destaca-se que em ambos os casos, os dados não são apenas de levantamentos fitossociológicos, mas de coletas gerais efetuadas e registradas em herbários.

Os dados apontados por este trabalho corroboram outros que destacam Fabaceae e Myrtaceae como famílias importantes dentro das florestas estacionais (Reitz et al. 1979; Klein 1972, 1978; Pennington et al. 2009; Ruschel et al. 2009; Rios et al. 2010). Gentry (1985) considera que ao lado de Fabaceae, Bignoniaceae é uma família importante no contexto das florestas estacionais, principalmente pela representatividade de lianas neste ambiente e cita a relevância de Myrtaceae nas florestas ombrófilas da margem Atlântica do Brasil, o que ressalta a contribuição também do contingente de espécies atlânticas nas florestas aqui descritas (Morellato \& Haddad 2000; OliveiraFilho \& Fontes 2000; Pennington et al. 2009). Particularmente, Myrtaceae é melhor representada nas florestas ombrófilas do que nas estacionais, tendendo a declinar em riqueza nos biomas cerrado e Amazônia (Oliveira-Filho \& Fontes 2000).

Sarmiento (1972) destaca, dentre os gêneros típicos das florestas estacionais Annona, Eugenia, Machaerium Pers., Myrcia, Pisonia L., Rauvolfia L. e Trichilia P. Browne. Todos figuram dentre aqueles mais representativos em número de registros por UA. 
Tabela 3 - Espécies arbóreas citadas por Reitz et al. (1979) para a Floresta Estacional Decidual, mas não encontradas pelo Inventário Florestal de Santa Catarina, fase III, com respectivas informações sobre ocorrência e raridade em Santa Catarina, com base na Flora Ilustrada Catarinense e também por Klein (1972).

Table 3 - Tree species cited for Seasonal Deciduous Forest (Reitz et al. 1979) but not sampled in Floristic Forest Inventory of Santa Catarina State, phase III, including information on their distribution and rarity in Santa Catarina based on Flora Ilustrada de Santa Catarina and Klein (1972).

\begin{tabular}{|c|c|c|}
\hline Família & Espécie & Observação \\
\hline Annonaceae & Annona neosalicifolia H.Rainer & Pouco frequente \\
\hline Aquifoliaceae & Ilex dumosa Reissek & Frequente \\
\hline Arecaceae & Euterpe edulis Mart. & Muito frequente \\
\hline \multirow[t]{2}{*}{ Bignoniaceae } & Cybistax antisyphilitica (Mart.) Mart. ex DC. & Muito rara \\
\hline & Handroanthus pulcherrimus (Sandwith) S.O. Grose & Muito rara \\
\hline Cardiopteridaceae & Citronella gongonha (Mart.) R.A.Howard & Muito rara \\
\hline Celastraceae & Maytenus muelleri Schwacke & Muito rara \\
\hline Clusiaceae & Garcinia gardneriana (Planch.\& Triana) Zappi & Rara \\
\hline Combretaceae & Terminalia australis Camb. & Muito frequente \\
\hline Cunnoniaceae & Weinmannia paulliniifolia Pohl ex Seringe & Raríssima \\
\hline \multirow[t]{3}{*}{ Euphorbiaceae } & Croton urucurana Baill. & Muito rara \\
\hline & Sebastiania schottiana Müll.Arg. & - \\
\hline & Pachystroma longifolium (Nees) I.M.Johnst. & Pouco frequente \\
\hline \multirow[t]{6}{*}{ Fabaceae } & Albizia polycephala (Benth.) Killip & Muito rara \\
\hline & Calliandra tweediei Benth. & Frequente \\
\hline & Inga sessilis (Vell.) Mart. & Frequente \\
\hline & Lonchocarpus guilleminianus (Tul.) Malme & Pouco frequente \\
\hline & Senna oblongifolia (Vog.) H.S.Irwin \& Barneby & - \\
\hline & Sweetia fruticosa Spreng. & Bastante rara \\
\hline \multirow[t]{2}{*}{ Lauraceae } & Ocotea acutifolia (Nees) Mez & Muito rara \\
\hline & Ocotea porosa (Nees) Barroso & rara \\
\hline Meliaceae & Guarea guidonia (L.) Sleumer & não ocorre em SC \\
\hline Monimiaceae & Mollinedia elegans Tul. & Muito rara \\
\hline Moraceae & Ficus cestrifolia Schott ex Spreng. & Bastante comum \\
\hline Myrtaceae & Eugenia florida DC. & Bastante rara \\
\hline \multirow[t]{4}{*}{ Myrtaceae } & Calyptranthes concinna DC. & Muito rara \\
\hline & Campomanesia eugenioides (Camb.) D.Legrand ex Landrum & Muito rara \\
\hline & Myrcia laruotteana Cambess. & Muito rara \\
\hline & Myrciaria tenella (DC.) O.Berg & Muito rara \\
\hline Rhizophoraceae & Erythroxylum microphyllum A.St.-Hil. & Muito rara \\
\hline Rubiaceae & Faramea porophylla (Vell.) Müll.Arg. & não ocorre em SC \\
\hline Salicaceae & Banara parviflora (A. Gray) Benth. & Rara \\
\hline \multirow[t]{2}{*}{ Sapotaceae } & Pouteria gardneriana (A. DC.) Radlk. & Muito rara \\
\hline & Pouteria salicifolia (Spreng.) Radlk. & Muito frequente \\
\hline \multirow[t]{2}{*}{ Solanaceae } & Brunfelsia uniflora (Pohl) D.Don & Frequente \\
\hline & Solanum rantonnei Carr. ex Lescuyer & Bastante rara \\
\hline
\end{tabular}

Por outro lado, Gentry (1995) cita Handroanthus (=Tabebuia Gomes ex DC.), Cordia L., Casearia Jacq., Bauhinia L., Trichilia, Erythroxylum P. Browne, Randia L., Hippocratea L., Serjania Mill., Croton L. e Zanthoxyllum L. como os gêneros mais amplamente representados nas florestas secas neotropicais. Muitos destes gêneros e, inclusive espécies, são também representados nas florestas ombrófilas em Santa Catarina, embora haja diferenças evidentes na representação de cada uma delas. Muitas destas diferenças foram discutidas por Gasper et al. (2012) que apontaram Apuleia leiocarpa, Rauvolfia sellowii, Bastardiopsis densiflora, Chrysophyllum gonocarpum, Cordia trichotoma, Holocalyx balansae, Myrocarpus frondosus e Pisonia ambigua como componentes de um conjunto de espécies que representa muito bem a estrutura fitossociológica das florestas estacionais catarinenses. 


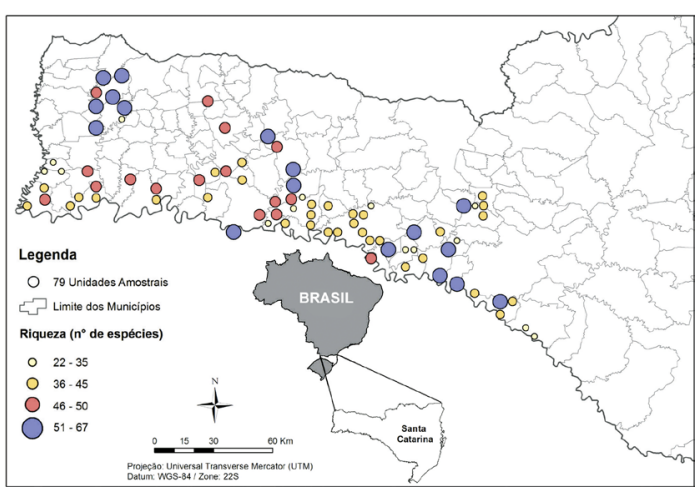

Figura 2 - Representação da distribuição geográfica da riqueza de espécies dos componentes arbóreo e arbustivo/subarbóreo em cada UA do IFFSC.

Figure 2 - Representation of species richness geographical distribution of arboreal and shrub/subarboreal components in each IFFSC sample unit.

Apesar da elevada riqueza registrada em Santa Catarina, 36 espécies arbóreas catalogadas por Reitz et al. (1979) na FED catarinense não foram encontradas, o que pode significar que as ações de supressão de florestas e sua simplificação constatada tiveram grande impacto sobre essas espécies, com forte indicação de extinção regional. Mas, apesar do esforço amostral desempenhado pelo IFFSC, algumas delas podem estar localizadas em diferentes áreas não estudadas, por isso considera-se relevante fazer novas pesquisas direcionadas para este conjunto indicado na Tab. 3 .

Segundo os dados de Vibrans et al. (2012b), comparativamente às demais regiões fitoecológicas do Estado, a FED possui um contingente florístico menor de espécies arbustivas/arbóreas (242 spp.) e compartilha menos espécies com as florestas ombrófilas (165) do que estas (Floresta Ombrófila Mista e Densa) compartilham entre si (202). A FED ainda possui um menor número de espécies exclusivas (19), contrastando fortemente com a Floresta Ombrófila Densa (339), embora menos com a Floresta Ombrófila Mista (57), o que certamente deve interferir na riqueza específica menor desta unidade. No total, foram amostradas 242 espécies, contra 452 na FOM e 736 na FOD.

De fato, estes números, bem como aqueles do presente trabalho, parecem concordar com os de Oliveira-Filho \& Fontes (2000), que atestam menor riqueza das florestas estacionais, definindo a FED em Santa Catarina como um conjunto empobrecido em espécies quando comparado às demais unidades tipologias florestais presentes no estado.
Gentry (1995) admite haver relação entre o volume anual de precipitações e a riqueza específica nas florestas tropicais situadas em baixas altitudes. Considerando que as florestas ora em análise estão situadas em regiões de elevado volume de precipitações anuais, com pequena tendência à estacionalidade (Wrege et al. 2002), estas tendem a ser mais ricas que outras áreas que abrigam florestas estacionais, mas que possuem marcante sazonalidade climática. Embora, a FED em Santa Catarina não esteja submetida a estacionalidade na precipitação, há diferenças marcantes entre as temperaturas médias de inverno e verão influenciadas pela diminuição do fotoperíodo no outono e inverno, e esses fatores desencadeiam deciduidade de folhas (Alberti et al. 2011) de mais de 50\% das espécies do dossel e emergentes (Klein 1972, 1978).

Portanto, analisando conjuntamente os dados deste trabalho e aqueles de outros autores, principalmente Gentry (1995) e Oliveira-Filho \& Fontes (2000), pode-se admitir que florestas estacionais de Santa Catarina, embora sejam classificadas como estacionais, representam algo como uma transição entre aquelas típicas de ambientes ombrófilos da margem atlântica e as estacionais, que tendem a predominar para oeste, fato este já discutido por Oliveira-Filho \& Fontes (2000).

O conjunto de dados apresentado neste trabalho revelou ainda dois aspectos importantes sobre a atual estrutura das florestas estacionais catarinenses: o primeiro é que a riqueza da flora arbórea/arbustiva é maior em fragmentos mais desenvolvidos do ponto de vista da sucessão ecológica (Fig. 3a). O segundo é que a dominância total é um bom preditor da riqueza de espécies arbóreas (Fig. 3b). Na verdade, ambas as conclusões combinam-se, pois é esperado que florestas em estádios mais avançados de sucessão apresentem estrutura mais complexa, evidenciado tanto maior riqueza, quanto abriguem maior biomassa, neste caso, medida indiretamente pela dominância total. O aumento da área basal representa maior proporção de cobertura do solo, refletindo em condições propícias ao estabelecimento de espécies de sub-bosque, ampliação do número de estratos e sinúsias não existentes, ou menos representativas, nos estágios iniciais e médios de sucessão florestal.

Em um contexto geral, as áreas de Floresta Estacional Decidual de Santa Catarina são razoavelmente ricas em espécies, se comparadas a outras florestas estacionais (Oliveira-Filho \& Fontes 2000; Liñares-Palomino et al. 2011), 

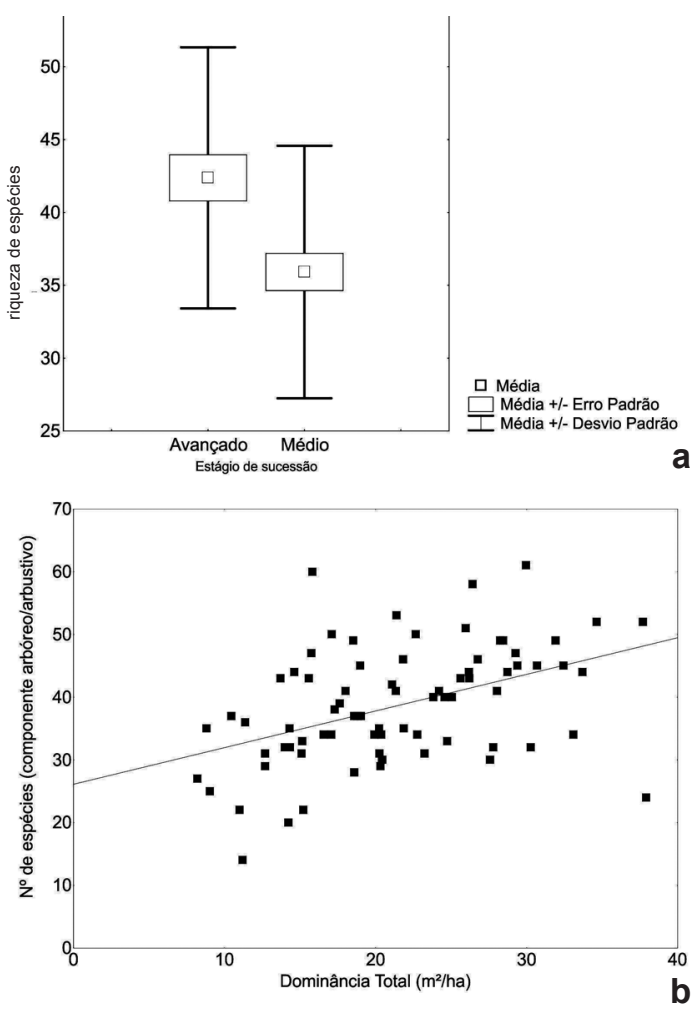

Figura 3 - a. Box-plot ilustrando os valores médios e a variação da riqueza do componente arbóreo de acordo com as duas categorias sucessionais; b. regressão linear entre a riqueza de espécies (S) e a dominância total (DoT, em $\mathrm{m}^{2} \cdot \mathrm{ha}^{-1}$ ). Conjunto de dados gerado pelos procedimentos do IFFSC.

Figure 3 - a. Box-plot depicting the richness variation of arboreal component according to successional categories; b. linear regression between species richness (S) and total dominance (DoT, $\mathrm{m}^{2} / \mathrm{ha}$ ). Data set were raised by means of IFFSC procedures.

provavelmente devido ao fato de ocuparem regiões com maior pluviosidade, mas apresentam famílias e gêneros amplamente característicos de florestas secas. Além disso, recebem acentuada influência florística oriunda das florestas ombrófilas situadas a leste. Além de menos diversas que as florestas ombrófilas, encontram-se mais acentuadamente fragmentadas e descaracterizadas que aquelas, indicando a necessidade de ações e políticas relacionadas à conservação.

\section{Agradecimentos}

Os autores agradecem à Fundação de Amparo à Pesquisa e Inovação do Estado de Santa Catarina, pelo financiamento do projeto Inventário Florístico
Florestal de Santa Catarina e aos proprietários das florestas amostradas. Agradecem também pela leitura e pelas valiosas críticas do professor Dr. João André Jarenkow e aos taxonomistas voluntários de diversas famílias botânicas pela identificação do material, em especial à equipe do Instituto de Pesquisas Jardim Botânico do Rio de Janeiro.

\section{Referências}

Alberti, L.F; Longhi, S.J. \& Morellato, P.C. 2011. Padrão fenológico de árvores e a relação com o clima em floresta estacional no Sul do Brasil. In: Schumacher, M.V.; Longhi, S.J.; Brum, E.J. \& Kilca, R.V. (orgs.). A floresta estacional subtropical: caracterização e ecologia no rebordo do Planalto Meridional. Santa Maria, Pallotti. Pp. 105-119.

APG. 2009. An update of the Angiosperm Phylogeny Group classification for the orders and families of flowering plants: APG III. Botanical Journal of the Linnean Society 161: 105-121.

Bigarella, J.J. 1964. Variações climáticas no Quaternário e suas implicações no revestimento florístico do Paraná. Boletim Paranaense de Geografia 10/15: 211-231.

Christenhusz, M.J.M.; Reveal, J.L.; Farjon, A.; Gardner, M.F.; Mill, R.R. \& Chase, M.W. 2011. A new classification and linear sequence of extant gymnosperms. Phytotaxa 19: 55-70.

Gasper, A.L. de; Uhlmann, A.; Vibrans, A.C.; Sevegnani, L. \& Meyer, L. 2012. Grupos florísticos da Floresta Estacional Decidual em Santa Catarina. In: Vibrans, A.C.; Sevegnani, L.; Gasper, A.L. \& Lingner, D.V. (eds.). Inventário florístico florestal de Santa Catarina. Vol. II. Floresta Estacional Decidual. Edifurb, Blumenau. Pp. 125-136.

Gentry, A.H. 1995. Diversity and floristic composition of neotropical dry forests. In: Bullock, S.H.; Mooney, H.A. \& Medina, E. (eds.). Seasonal dry tropical forests. Cambridge University Press, Cambridge. Pp. 146-194.

IBGE. 1992. Manual técnico da vegetação brasileira. IBGE, Rio de Janeiro.

Klein, R.M. 1972. Árvores nativas da Floresta Subtropical do Alto Uruguai. Sellowia 24: 9-62.

Klein, R.M. 1978. Mapa fitogeográfico de Santa Catarina. In: Reitz, R. (ed.). Herbário Barbosa Rodrigues, Itajaí. 24p.

Leite, P.F. \& Klein, R.M. 1990. Geografia do Brasil. Vol. 2. Região Sul. Rio de Janeiro, IBGE.

Linares-Palomino, R.; Oliveira-Filho, A.T. \& Pennington, R.T. 2011. Neotropical seasonally dry forests: diversity, endemism and biogeography of woody plants. In: Dirzo, R.; Young, H.S.; Mooney, H.A. \& Ceballos, G. (eds.). Seasonally dry tropical forests: ecology and conservation. Island Press, Washington, D.C. Pp. 3-21. 
MMA (Ministério do Meio Ambiente). 2008. Instruçãoo Normativa $n^{\circ} 6$, de 23 de setembro de 2008. Lista oficial das espécies da flora brasileira ameaçadas de extinção. Diário Oficial da República Federativa do Brasil, Brasília, Df, 24 set. 2008. Seção 1. Pp. 75-83

Meyer, L.; Vibrans, A.C.; Gasper, A.L.; Lingner, D.V. \& Sampaio, D. K. 2012. Espécies exóticas encontradas nas florestas de Santa Catarina. In: Vibrans, A.C.; Sevegnani, L.; Gasper, A.L. de \& Lingner, D.V. (eds.). Diversidade e conservação dos remanescentes florestais. Vol. I. Edifurb, Blumenau. Pp. 236-265.

Morellato, L.P.C. \& Haddad, C.F.B. 2000. Introduction: The Brazilian Atlantic Forest. Biotropica 32: 786-792.

Oliveira-Filho, A.T. \& Fontes, M.A.L. 2000. Patterns of floristic differentiation among Atlantic forests in southeastern Brazil and the influence of climate. Biotropica 32: 793-810.

Pennington, R.T.; Prado, D.A. \& Pendry, C. 2000. Neotropical seasonally dry forests and Pleistocene vegetation changes. Journal of Biogeography 27: 261-273.

Pennington, R.T.; Lavin, M. \& Oliveira-Filho, A.T. 2009. Woody plant diversity, evolution, and ecology in the tropics: perspectives from seasonally dry tropical forests. Annual Review of Ecology, Evolution, and Systematics 40: 437-457.

Prado, D.E. \& Gibbs, P.E. 1993. Patterns of species distributions in the dry forests of South America. Annals of the Missouri Botanical Garden 80: 902-927.

Reitz, P.R. 1965. Plano de coleção. In: Reitz, R. (ed). Herbário Barbosa Rodrigues, Itajaí. 71p.

Reitz, R.; Klein, R.M. \& Reis, A. 1979. Projeto Madeira Santa Catarina. Lunardelli, Florianópolis. 320p.

Ribeiro, M.C.; Metzger, J.P.; Martensen, A.C.; Ponzoni, F.J. \& Hirota, M.M. 2009. The Brazilian Atlantic Forest: How much is left, and how is the remaining forest distributed? Implications for conservation. Biological Conservation 142: 1141-1153.

Ríos, R.C.; Galvão, F. \& Curcio, G.R. 2010. Estructura de las principales especies arbóreas en el Parque Cruce Caballero y su similitud floristica con areas de Argentina y de Brasil. Ciência Florestal 20: 193-206.

Ruschel, A.R.; Guerra, M.P. \& Nodari, R.O. 2009. Estrutura e composição florística de dois fragmentos da Floresta Estacional Decidual do Alto-Uruguai, SC. Ciência Florestal 19: 225-236.
Sarmiento, G. 1972. Ecological and floristic convergences between seasonal plant formations of tropical and subtropical South America. Journal of Ecology 60: 367-410.

Sevegnani, L.; Uhlmann, A.; Gasper, A.L. de; Vibrans, A.C.; Stival-Santos, A.; Verdi, M. \& Dreveck, S. 2013. In: Vibrans, A.C.; Sevegnani, L.; Gasper, A.L.; Lingner, D.V. (eds.). Estádios sucessionais da Floresta Ombrófila Mista em Santa Catarina. Vol. III. Floresta Ombrófila Mista. Edifurb, Blumenau. Pp. 255-271.

Stehmann, J.R.; Forzza, R.C.; Salino, A.; Sobral, M.; Costa, D.P. \& Kamino, L.H.Y. 2009. Plantas da Floresta Atlântica. Instituto de Pesquisas Jardim Botânico do Rio de Janeiro, Rio de Janeiro.

Thiers, B. 2012. Index Herbariorum: a global directory of public herbaria and associated staff. New York Botanical Garden's Virtual Herbarium

Vibrans, A.C.; McRoberts, R.E.; Lingner, D.V.; Moser, P. \& Nicoletti, A. 2012a. Extensão original e remanescentes da Floresta Estacional Decidual em Santa Catarina. In: Vibrans, A.C.; Sevegnani, L.; Gasper, A.L. \& Lingner, D.V. (eds.). Inventário Florístico Florestal de Santa Catarina, Vol. II, Floresta Estacional Decidual. Blumenau, Edifurb. Pp. 25-31.

Vibrans, A.C.; Moser, P.; Lingner; D.V. \& Maçaneiro, J.P. 2012b. Análise estatística do IFFSC e estimativas dendrométricas. In: Vibrans, A.C.; Sevegnani, L.; Gasper, A.L. \& Lingner, D.V. (eds.). Inventário florístico florestal de Santa Catarina. Vol. I. Diversidade e conservação dos remanescentes florestais. Edifurb, Blumenau. Pp. 79-95.

Vibrans, A.C.; McRoberts, R.E.; Moser, P. \& Nicoletti, A. 2013. Using satellite image-based maps and ground inventory data to estimate the area of the remaining Atlantic forest in the Brazilian state of Santa Catarina. Remote Sensing of Environment 130: 87-95.

Vibrans, A.C.; Sevegnani, L.; Lingner, D.V.; Gasper, A.L. \& Sabbagh, S. 2010. Inventário florístico florestal de Santa Catarina (IFFSC): aspectos metodológicos e operacionais. Pesquisa Florestal Brasileira 30: 291-302.

Wrege, M.S.; Steinmetz, S.; Reisser-Junior, C. \& Almeida, I.R. 2011. Atlas climático da Região Sul do Brasil: estados do Paraná, Santa Catarina e Rio Grande do Sul. Embrapa Clima Temperado/ Embrapa Florestas, Pelotas, Colombo. 336p. 